IV. Absolute Hürtemessung; von F. Auerbach. (Der Kgl. Gesellschaft d. Wiss. zu Göttingen im Auszuge vorgelegt an 6. Dec. 1890.)

(Hierzu Tar. 1 B Fig. 1-2.)

Eine der auffälligsten und wichtigsten Eigenschaften der festen Körper ist ihre Härte. In der That ist die Zahl der Bemühungen, das Härteproblem oder in ihm enthaltene einzelne Aufgaben zu lösen, eine überaus grosse, und es sind dabei zum Theil sehr interessante Erscheinungen und Ergebnisse zu Tage getreten. Trotzdem muss man sagen, dass in Bezug auf die Hauptaufgabe der Erfolg bis auf den heutigen Tag ein negativer geblieben ist. Das Problem, welches eine physikalische Eigenschaft der Körper uns darbietet, zerfällt nämlich in drei Theilprobleme: die wissenschaftliche Definition des betreffenden Begriffs, wonach dieser den Charakter einer mathematischen Grösse erhălt; die Auffindung einer Methode, resp. die Construction eines Apparates, um die so definirte Grösse zu messen, endlich die wirkliche Ausführung derartiger Bestimmungen für möglichst viele,Körper und unter möglichst verschiedenen Bedingungen. Dabei kommt es zunächst durchaus nicht auf die Einfachheit und praktische Brauchbarkeit des Verfahrens an - diese Frage gehört einem viel späteren Stadium der Untersuchung an es handelt sich wesentlich um die Schaffung einer theoretischen Grundlage und um den erfahrungsmässigen Nachweis, dass auf dieser Grundlage das experimentelle Gebäude errichtet und das Ziel mit befriedigender Annäherung erreicht werden kann. Bis vor wenigen Jahren war nun in Bezug auf die Härte keines der angeführten drei Theilprobleme gelöst; erst Hertz gelang dies hinsichtlich des ersten Theilproblems, indem er auf Grund böchst scharfsinniger Betrachtungen zu einer Definition gelangte, welche mit der allgemeinen Vorstellung und den fruheren Definitionen in allem Wesentlichen und Nothwendigen übereinstimmt, von ihren principiellen Fehlern, ihren Lücken und ihrem überflüssigen Beiwerk jedoch frei ist. Ich glaube nun, dass es mir gelungen ist, auf Grund dieser Definition ein Verfahren zu ermitteln, welches, bis auf einen bestimmten, noch weiterer 
Aufklärung bedürftigen Punkt, theoretisch und experimentell befriedigende Ergebnisse liefert. Demgemäss enthält:

$\$ 1$ eine Uebersicht über die bisherigen Untersuchungen,

$\$ 2$ die Theorie, soweit sie in Betracht kommt,

$\$ 3$ die Methode im allgemeinen,

$\$ 4$ die Beschreibung des Apparats,

$\$ 5$ allgemeines über die Beobachtungen,

$\$ 6$ die Constanten und Fehlerquellen,

$\$ 7$ die experimentelle Prüfung der Theorie,

$\S 8$ die Messung der Elasticität und der Härte für einige Substanzen.

Hinsichtlich des letzten Punktes sei jedoch ausdrücklich bemerkt, dass es sich hier lediglich um Angaben handelt, welche zeigen sollen, welche Brauchbarkeit und Genauigkeit dem Verfahren zukommt, in wie weit also das zweite der genannten Theilprobleme als gelőst zu betrachten ist. Systematische, das dritte Theilproblem erleảigende Härtemessungen, sowie zahlreiche, bei der Untersuchung auftauchende weitere Fragen, die zum Theil im Texte schon angedeutet sind, müssen späteren Arbeiten vorbehalten bleiben. ${ }^{3}$ )

\$1. Bisherige Untersuchungen.

Man kann sich hinsichtlich der Definition und Bestimmung irgend einer Grösse, also auch der Härte, drei verschiedene Ziele stecken. Man kann erstens sich damit begnügen, festzustellen, dass die Härte in einem bestimmten Falle grösser ist, als in einem bestimmten anderen, und man kann hiernach die Körper in eine. Reihe ordnen, in welcher jeder folgende Körper härter ist, als der vorhergehende. Den einzelnen Gliedern der Reihe kann man alsdann Nummern beigeben; aber diese Nummern werden selbstverstandlich nicht die Bedeutung von Zahlen haben. Ueberdies muss, um auch nur die Berechtigung der Nummern zu sichern, vorerst untersucht werden, 1) ob stets, wenn $B$ härter als $A$, auch $A$ weniger hart als $B$ ist, 2) ob, wenn $C$ härter als $B$ und $B$ härter als $A$, auch stets $C$ härter als $A$

1) Die Beobachtungen wurden in den Räumen und mit den Mitteln des hiesigen physikalischen Instituts ausgeführt, dessen Director, Hrn. Prof. Winkelmannn, ich auch an dieser Stelle meinen besten Dank sage. 
ist. Für manche physikalische Eigenschaften ist dies bekanntlich nicht der Fall, oder mindestens nicht für alle Körper, und es ist dann eben nicht möglich, alle Körper in eine einzige derartige Reihe einzuordnen. Erst wenn diese Möglichkeit festgestellt ist, ist es im Princip möglich, den zweiten Schritt zu machen, nämlich die Härtenummern durch Härtezahlen zu ersetzen, d. h. durch Zahlen, deren Verhältnise für irgend zwei Körper ihr Härteverhältniss darstellt. Man erhält dann ein relatives Maass der Härte, dessen Einheit willkürlich gewählt wird, etwa dadurch, dass man die Härte eines bestimmten Körpers gleich 1 setzt. Aber auch hier ist wieder zu bedenken, dass die Zahlen sehr wohl nicht blos absolut, sondern auch in ihren Verbältnissen verschieden ausfallen, je nach der Wahl der Methode. Man kann endlich drittens zum absoluten Maass der Härte übergehen, d. h. sio für jeden Körper unabhängig von anderen Körpern oder einem Normalkörper durch absolute Grundeinheiten ausdrücken.

Die bekannteste und noch heute am meisten benutzte Definition und Methode, die des Ritzens, wonach ein Körper härter ist, als ein anderer, wenn er, in Spitzenform angewandt, eine ebene Fläche dieses anderen ritzt, liefert nur Härtenummern. Von den beiden, eine Scala ermöglichenden Voraussetzungen ist die erstere näherungsweise erfüllt, nämlich dann, wenn der Härteunterschied der beiden Körper ein beträchtlicher ist. Ist er nur gering, so beobachtet man dagegen sehr häufig, dass jeder von ihnen, in Spitzenform angewandt, eine ebene Fläche des anderen ritzt; man hilft sich alsdann damit, diesen Widerspruch auf Rechnung der Empfindlichkeit der Methode zu setzen, indem man sagt, diese beiden Körper seien gleich hart. Da auch die zweite der obigen Voraussetzungen in den bisher untersuchten Fällen sich als erfüllt erwiesen hat, steht der Aufstellung einer Reihe von beschränkter Genauigkeit nichts im Wege. Der erste, welcher dies that, Ha u y, begntigte sich mit vier Stufen, deren Grenzen Kalkspath, Glas, Quarz bilden. Mohs ging bis zu zehn Stufen hinauf; und obgleich spätere Mineralogen noch einige $Z$ wischenstufen einschalteten, weil es den Anschein hatte, als wären einige der Mohs'schen Stufen sehr beträchtlich grösser als andere, so ist es doch bis zum heu. 
tigen Tage bei der Mohs'schen Scala geblieben, und zwar mit Recht, da für die auch nur annähernd gleiche Grösse der Stufen gar kein Anhalt vorliegt, und bei dem angeführten Mangel der Grundlagen eine weitere Verkleinerung der Stufen weit eher zu Fehlern als zu grösserer Genauigkeit führen würde.

Den Uebergang zu messenden Versuchen machte Fran. kenheim ${ }^{1}$, indem er den Druck schätzte, den er mit der Hand ausüben musste, um mit einer bestimmten Spitze in der zu prüfenden Fläche einen Ritz zu erzeugen. Aber erst Seebeck ${ }^{2}$, Franz ${ }^{3}$, Grailich und Pekàrek${ }^{4}$ ), F. Exner ${ }^{6}$ ), Pfaff ${ }^{\boldsymbol{\theta}}$ ), Turner ${ }^{7}$ ) benutzten Apparate, welche Zahlenwerthe für diesen Druck, resp. für die bei bestimmtem Druck erzielte Tiefe des Eindringens anzugeben gestatteten und den Namen Sklerometer erhielten. Zunächst ist zu bemerken, dass, wie übrigens Exner selbst zugibt, eine wirkliche Messungsmethode hiermit gar nicht gewonnen ist. Denn eine Messung schliesst den wahren Werth der zu messenden Grösse in zwei Grenzen ein, von deren Abstand die Genauigkeit der Messung abhängt. Vorliegende Methode liefert aber nur eine obere Grenze; die Feststellung der unteren Grenze bleibt der Schätzung überlassen. Einen Fortschritt gegenüber der Frankenheim'schen Methode bedeutet die Seebeck'sche nur insofern, als sie die Schätzung ron der Hand auf das Auge allein überträgt, das viel empfindlicher im Schätzen ist. ${ }^{8}$ ) Aber, hierron abgesehen, sind gegen diese Definition und Methode vor allem zwei Einwände zu machen.

1) Frankenheim, De cohaesione etc. Inaug.-Diss. Breslau 1829.

2) S eebeck, Progr. Cöln. Real-Gymn. 1833.

3) Franz, De lapidarum duritate. Inaug.-Diss. Bonn 1850. Pogg. Ann. 80. p. 37. 1850.

4) Grailich u. Pekárek, Wien. Ber. 13. p. 410. 1854.

5) F. Exner, Unters. üb. d. Härte an Krystalleüchen. Wien 1873.

6) Pfaff, Münchn. Ber. 1883. p. 55 u. 372. Wie P faff dazu kommt, in seinen Arbeiten stets von absoluter Härte zu reden, da es sich doch im günstigsten Falle (s. u.) um die relative handelt, ist mir unerfindlich, da doch der Ausdruck "absolut" in Bezug auf Messen eine ganz bestimmte Bedeutung hat.

7) Turner, Proc. Birm. Phil. Soc. 5. (2) 1887.

8) Exner sagt: „Der grosse Untersehied zwischen dieser Methode und einer Messung wird jedem deutlich, der sich mit ibr beschäftigt." 
Erstens ihre sehr specielle Bedeutung, d. h. die Abhängigkeit des Resultats ron zahlreichen speciellen Umständen. Speciell ist dabei zunächst das Material der Spitze, und dieses Bedenken wiegt um so schwerer, als das hierfür geeignetste Material, Stahl, das auch meist benutzt wird, nur schwer exact definirt, also kaum dafür gesorgt werden kann, dass bei verschiedenen Apparaten resp. Messungen Spitzen von gleichartigem Stahl Verwendung finden, wozu noch kommt, dass es nicht angängig ist, für alle Körper, weiche wie harte, dasselbe Ritzmaterial zu benutzen, weil sehr weiche unter einer sehr harten Spitze unmessbar kleine Drucke erfordern, harte aber nur durch eine sebr harte Spitze geritzt werden. So benutzte Franz für weniger harte Körper eine Stahlspitze, für härtere eine Diamantspitze, die Continuität stellte er her, indem er für zwei Körper Messungen mit beiden Spitzen ausführte. Die Hărtezahlen dieser beiden Stoffe stehen nun allerdings bei Benutzung der beiden Spitzen in ziemlich genau gleichem Verhältniss; es wäre aber durchaus voreilig, zu schliessen, dass dies immer und insbesondere auch bei Körpern von grösserem Härteunterschied der Fall sein müsste. Speciell ist zweitens die Form der Spitze, also im wesentlichen der Grad ihrer Spitzigkeit; in den genann. ten Publicationen ist hieruber nichts gesagt, und er würde sich auch kaum mit Genauigkeit angeben lassen. Nun ist es aber zweifellos, nicht nur, dass bei verschiedenen Apparaten die Spitzen sehr verschieden scharf waren und sind, sondern auch, dass sich bei einem und demselben Apparat die Spitze mit der Zeit abnutzt. Da es einleuchtend ist, dass hierdurch das Resultat ganz wesentlich beeinflusst werden muss, so folgt, dass die Messungen uberhaupt nicht miteinander vergleichbar sind. Drittens ist es die Art und Weise des Ritzens, welche eine sehr verschiedene sein kann, namentlich hinsichtlich der Geschwindigkeit der Bewegung und der Richtung des dabei ausgeübten Druckes, wie denn auch einige der genannten Beobachter Angaben über die geeignetste Geschwindigkeit und die passendste Stellung der Spitze machen. Dass der Einfluss der Geschwindigkeit unter Umstanden bis zu einer die ganze Erscheinung umkehrenden Höhe anschwellen kann, zeigt die zuerst von Barnes und Ann. d. Phys. u. Chem. N. F. XIIII. 
Perkins und dann wiederholt festgestellte Thatsache, dass die Randfläche eines schnell rotirenden Körpers, an den eine Feile oder ein Grabstichel angelegt wird, unversehrt bleibt, ja sogar die letztgenannten Körper erheblich angreift, selbst wenn jener ganz beträchtlich weicher ist, als diese, wobei nähere Versuche lehren, dass es sich nicht um thermische oder andere Nebeneinflüsse, sondern um den directen Ein. fluss der Bewegung handelt; die Geschwindigkeit braucht übrigens, wie Inan mit der Zeit fand, durchaus nicht sehr gross zu sein - noch bei ein bis zwei Meter in der Secunde kann man das härteste Gusseisen mit stählernen Werkzeugen abdrehen.

Diese Betrachtungen führen naturgemäss auf die für unseren Gegenstand principiell wichtigste Frage, ob nämlich die rorliegende Definition im Princip annehmbar sei. Diese Frage muss entschieden verneint werden. Die bisher be. sprochenen Uebelstände treten dabei, so wichtig sie auch sein mögen, doch zurück hinter den Umstand, dass die durch Ritzen definirte Härte ein viel zu verwickelter Begriff ist, als dass Aussicht vorhanden wäre, auf ihr als Basis das Härteproblem zu lösen. Und zwar liegt die Complication wesentlich in dem in die Härte hineingelegten, in ihr aber nicht nothwendig liegenden Bewegungsvorgang, insbesondere der beim Ritzen stattfindenden lateralen Bewegung. Man kann sich auch sehr wohl vorstellen, wie man hierauf verfallen ist; man wird ursprünglich untersucht haben, ob die prüfende Spitze einen punktförmigen Eindruck in das zu prüfende Material macht, und nur, weil dieser sich schlecht erkennen lässt, wird man $z u$ linienhaftem Eindruck übergegangen sein. Diese Vermuthung findet ibre Bestätigung in der Thatsache, dass schon lange vor der dynamischen die statische Methode der Härtemessung existirt hat. Da nun, wie sich zeigen wird, die statische Definition ausreicht, um die Härte als eine ganz charakteristische, selbständige und einfach zu verstehende Eigenschaft der Körper zu definiren, so ist alles weitere von Uebel. Damit soll durchaus nicht in Abrede gestellt werden, dass gerade die Methode des Ritzens interessante Ergebnisse geliefert hat, z. B. die Verschiedenheit der Härte einer und derselben Krystall- 
fläche beim Ritzen in verschiedenen Richtungen; zu einem Verstandniss dieser Erscheinungen wird man aber am besten gelangen, wenn man zunächst das statische Härteproblem löst und dann zusieht, welche Modificationen des so ermittelten Verhaltens eintreten, falls man von der eigentlichen Härte zum Widerstand gegen Ritzen ubergeht. Auch in praktischer Hinsicht wird die Methode des Ritzens, z. B. zu raschen Interpolationsmessungen, von Werth bleiben, da sie sich durch Bequemlichkeit auszeichnet und in Fällen, wie der genannte, fehlerbafte Ergebnisse alsdann kaum noch zu befürchten sein werden. ${ }^{1}$ )

Unter den bezeichneten Umständen ist es als ein Fortschritt zu bezeichnen, dass die statische Methode - statisch, insofern laterale Bewegungen ausgeschlossen sind - in neuerer Zeit von verschiedenen Seiten wieder aufgenommen worden ist; insbesondere sind hier die Arbeiten von Crace-Calvert und Johnson, Hugueny ${ }^{2}$ ), Botton $\theta^{3}$ ) und wiederum Pfaff ${ }^{4}$ ) zu nennen. Man schlägt, bohrt oder presst eine Spitze in das zu untersuchende Material ein und definirt die Härte entweder als das zur Erreichung einer bestimmten Tiefe erforderliche Belastungsgewicht, oder als die hierzu bei bestimmtem Druck nöthige Zanl von Umdrehungen des Bohrers (Mesosklerometer von Pfaff), oder als die Tiefe dieses Eindringens bei gegebener Belastung, oder endlich als die Zeit, welche bei gegebener Belastung erforderlich ist, um bei gleichförmigem Eindringen eine bestimmte Tiefe zu erreichen. Man ersieht aber schon aus dieser Zusammenstellung, dass hier verschiedene Momente ineinander greifen und zwar in einer nicht allgemein anzugebenden Weise; auch müssen dabei Annahmen betreffend die Proportionalität der genannten Grössen mit der Härte gemacht werden, welche zum Theil vielleicht nicht einmal angenähert richtig

1) Hugueny (s. u.), weicher auch schon derartige Betrachtungen angestellt hat, stellt schliesslich drei verschiedene „Härten“ auf, eine „tangentiale" und zwei "normale".

2) Hugueny, Recherches exp. sur la dureté des corps. Paris 1865. (Auszug in den Ber. d. Strassb. Ges. vom gleichen Jahre.)

3) Bottone, Sill. Journ. 1873, p. 457. - Pogg. Ann. 150. p. 644. 1873.

4) Pfaff, Münchn. Ber. 1884. p. 255. 
sind. Aber noch wichtiger ist der Einwand, dass bei der hier gekbbten Behandlungsweise das betreffende Material nicht nur sehr stark beansprucht - das ist bei einer Härteuntersuchung naturlich nicht zu vermeiden - sondern an der zu untersuchenden Stelle geradezu zerstört wird, dass man also in dem Zeitpunkte, wo man die Härte misst, schon längst nicht mehr den ursprünglichen Körper vor sich hat. Es ergibt sich also, dass auch diese Methoden keine brauchbaren Ergebnisse liefern können.

Zur thatsächlichen Bekräftigung des in Obigem enthaltenen absprechenden Urtheils habe ich aus der bezüglichen Literatur zwei der wenigen grösseren Zahlenreihen herausgegriffen, welche einen Vergleich zulassen, nämlich diejonige von Franz, und die von $P$ faff in seiner letzten Abhandlung mitgetheilte, mit dem Mesosklerometer erhaltene; in der ersten Tabelle sind die Zahlen für Gyps, in der zweiten die für Korund einander gleich gesetzt. Die beiden Zahlen für die Härte eines und desselben Materials stehen, wie man sieht, dort in einem bis zu 15 hinauf-, hier in einem bis zu 2,3 hinauf- und 0,2 hinabgehenden Verbältniss. ${ }^{1}$ )

Gyps gleichgesetzt.

\begin{tabular}{l||r|r|r}
\hline Stoff & Franz & Pfaff & $\begin{array}{c}\text { Verhält- } \\
\text { niss }\end{array}$ \\
\hline \hline Gyps & 6 & 6 & 1 \\
Kalkspath & 36 & 8 & $4^{1 / 1}$ \\
Flussspath & 144 & 20 & 7 \\
Apatit & 652 & 38 & 15 \\
Feldspath & 1040 & 105 & 10 \\
Quarz & 1770 & 160 & 11 \\
Topas & 2230 & 240 & 9 \\
Korund & 2650 & 340 & 8
\end{tabular}

Kor und gleichgesetzt.

\begin{tabular}{l||r|r|r}
\hline Stoff & Franz & Pfaff & $\begin{array}{c}\text { Verh\&lt- } \\
\text { niss }\end{array}$ \\
\hline Korund & 340 & 340 & 1,0 \\
Topas & 298 & 240 & 1,2 \\
Quarz & 228 & 160 & 1,4 \\
Feldspath & 134 & 105 & 1,3 \\
Apatit & 84 & 38 & 2,2 \\
Flusspath & 19 & 20 & 0,9 \\
Kalkspath & 5 & 8 & 0,6 \\
Gyps & 1 & 6 & 0,2
\end{tabular}

Das Verdienst, eine Definition der Härte aufgestellt zu haben, welche, ohne dem damit verbundenen Begriffe untreu zu werden, doch alle Mängel und Unklarheiten der früheren Definitionen vermeidet, gebührt Hertz. ${ }^{2}$ ) Erstens ersetzt

1) Aehnlich kolossale Differenzen ergeben für Metalle die Reihen von Bottone und von Hugueny (Kupfer $=100$, Nickel 104 resp. 58, Platin 81 resp. 150, Blei 42 resp. 9 u. s. w.).

2) H. Hertz, Verh. Berl. physik. Ges. 1882. p. 67 u. Verb. d. Ver. z. F. d. Gewerbfl. 1882. p. 441. 
er die bisher stets zur Prüfung benutzte Spitze durch eine kugelförmige Endfläche oder, richtiger gesagt - da doch die Spitze nichts anderes als eine derartige Kugelfläche mit sehr kleinem Radius ist - er gibt ibr eine nicht sehr kleine, sonst beliebige, aber genau messbare Krümmung; zweitens lässt er dahingestellt, aus welchem Material die Spitze bestehe, derart, dass man hierfür, wenn man es für wlunschenswerth erachtet, auch denjenigen Stoff wählen kann, aus welchem der zu untersuchende Körper besteht; das Ergeb. niss hängt alsdann überbaupt von keinem fremden Material $a b$; drittens endlich bringt er dem zu untersuchenden Körper keine Verletzung von bestimmtem Grade bei, sondern lässt ihn nur eben seine Elasticitätsgrenze erreichen. Hiernach lautet die Definition der Härte folgendermaassen: Die Härte ist die Elasticitätsgrenze eines Körpers bei Berührung einer ebenen Fläche desselben mit einer kugelförmigen Flăche eines anderen Körpers. Damit ist zugleich der Begriff der Härte eingereiht unter die ubbrigen analogen Begriffe, welche sich auf die Vorgänge des Zuges, der Biegung u. s. w. beziehen. Es kommt nun freilich darauf an, theoretisch zu ermitteln, wie sich bei der Berührung zweier Körper die Druck- und Oberflächenverhältnisse gestalten, da hiervon die Beanspruchung des Materials und somit die Lage der Elasticitätsgrenze abhängen wird. Durch die Lösung dieses Problems hat $\mathrm{Hertz}{ }^{1}$ ) die Grundlage für exakte Härtemessung im Princip sichergestellt. Weniger erfolgreich war er bei seinen Versuchen, eine geeignete Methode ausfindig zu machen und wirkliche Härtemessungen vorzunehmen, sodass or sie sehr bald aufgab. Die einzigen von ihm ermittelten Zahlen beziehen sich auf Glas, und 'zwar erhielt er für die Härte desselben

durch Pressen einer harten Stahllinse gegen Spiegelglas . $135 \mathrm{~kg} / \mathrm{qmm}$ aus dem Zusammenstoss zweier Glaskugeln . . . . . . 150 " durch Zusammenpressen zweier dünner Glasstäbe . . . 190 "

Diese Zahlen sind allerdings erheblich verschieden, und es dürfte nach meinen Erfahrungen nicht zulässig sein, mehr als einen kleinen, etwa den dritten oder vierten Theil der

1) H. Hertz, Crelle's Journ. 92. p. 156. 1882. 
Differenzen durch die vermuthliche Verschiedenheit der geprüften Glassorten zu erklären. Es bleiben also in der That Differenzen bestehen; auf ihre Erklärung kann jedoch hier um so weniger eingegangen werden, als die Zahl der Versuche im Verhältniss zur Mannigfaltigkeit der benutzten Körperformen eine zu kleine ist und Angaben über die Dimen. sionen der Körper, sowie luber das Detail der gemessenen Erscheinungen nicht gemacht sind.

\section{§2. Theorie.}

Die drucklose Berührung einer Ebene mit einer Kugelfläche findet in einem Punkte statt. Wird jetzt ein bestimmter Normaldruck ausgeübt, so verändern sich beide Flächen innerhalb eines gewissen Bereichs, die Ebene krümmt sich, die Kugelfläche plattet sich bis $z$ einem gewissen Grade ab, und damit geht der Berührungspunkt in eine beiden Körpern gemeinsame Fläche über. Diese Fläche heisst die Druckfläche; sie ist weder eben, noch von der Krümmung der Kugelfläche, ihre Krümmung liegt vielmehr zwischen beiden Werthen; wie gross sie ist, hängt nicht nur von der Krümmung der Kugelfläche, sondern auch von den Elasticitätsverhältnissen der beiden Körper ab; begrenzt endlich ist die Druckfläche durch eine Kreislinie.

Wird der ausgeübte Druck gesteigert, so nimmt die Druckfläche an Grösse zu, und der gesteigerte Druck vertheilt sich somit auf eine grössere Fläche. Nun hängt die Beanspruchung des Materiales offenbar nicht von dem ausgeübten Gesammtdruck, sondern von dem Druck auf die Flächeneinheit ab, und es kommt daher darauf an, wie sich diese letztere Grösse bei Steigerung des Gesammtdruckes verhält, resp. nach welchem Gesetze sie selbst wächst - denn dass auch der Druck pro Flächeneinheit wächst, folgt schon aus den bekanntesten Erfahrungsthatsachen. Nach welchem Gesetze der Einheitsdruck mit dem Gesammtdrucke zunehmen wird, hängt von dem Gesetze $a b$, nach welchem die Druckfäche mit dem Gesammtdrucke wächst. Die Theorie zeigt nun, dass der Radius der Druckfläche wie die Kubikwurzel aus dem Gesammtdruck wächst, also die Druckfläche selbst wie die $2 / 3$. Potenz desselben; so viel also geht durch 
Vertheilung verloren, und der Einheitsdruck steigt nur wie die Kubikwurzel aus dem Gesammtdruck. Auch die Frage, wie sich der Gesammtdruck auf die Fläche vertheilt, wird von der Theorie beantwortet, und zwar dahin, dass der Druck zu einer bestimmten Zeit rom Mittelpunkte der Druckfläche aus, wo er am grössten ist, nach dem Rande hin, wo er Null ist, allmählich und zwar nach dem Gesetze $\sqrt{1-x^{2}}(x$ der Bruchtheil des Druckflächenradius, um welchen die betreffende Stelle vom Mittelpunkte absteht), abnimmt; der oben schlechthin als solcher bezeichnete Einheitsdruck hat also nur die Bedeutung eines Durclischnittswerthes, der im Mittelpunkt stattfindende Maximalwerth verbält sich zu ihm wie 3:2. Wächst nun der Gesammtdruck mehr und mehr, so wird auch der letztgenannte Maximaldruck inmer grösser, und bei einem bestimmten Werthe desselben wird der eine der beiden Körper oder werden beide, falls sie aus demselben Stoffe bestehen, die Elasticitätsgrenze erreichen, was sich darin zeigen wird, dass bei einem plastischen Körper eine dauernde Deformation eintritt, also eine Deformation, die auch nach Aufhebung des Druckes bestehen bleibt, dass dagegen bei einem spröden Körper der Zusammenhang der Theile an gewissen Stellen, also durch einen Sprung, aufgehoben wird, Dieser Grenzwerth des im Mittelpunkte der Druchfäche in normaler Richtung herrschenden Einheitsdruckes ist nach der Definition von Hertz die Härte des letreffenden Körpers. Ausser dem Normaldruck herrschen in jedem Punkte natürlich auch seitliche Drucke, und man kann dieselben wenigstens im grossen einigermaassen verfolgen. Im Mittelpunkte der Druckfläche sind sie positiv, d. h. hier findet allseitige Com. pression des Materiales statt, woraus für unsere Frage hervorgeht, dass hier ein Sprung nicht zu erwarten ist. Wobl aber ist dies in der Nachbarschaft des Randes der Druckflache der Fall, da hier der seitliche Druck negativ, d. b. vom Charakter eines Zuges ist. $\mathrm{Da}$ aus Symmetriegründen überdies die Kreisform folgt, so werden wir einen kreisförmigen, die Druckfläche umschliessenden Sprung erhalten.

Bisher wurde ein bestimmtes System zweier sich berührender Körper angenommen. Es entsteht jetzt die Frage, wie sich die Verhältnisse ändern, wenn es durch ein anderes, 
in irgend einer Weise von jenem abweichendes, ersetzt wird. Die Abweichung kann im wesentlichen nur zwei Punkte betreffen; es kann nämlich 1) die Kugelfläche eine andere Krümmung haben, und es können 2) die Körper aus Stoffen anderer Elasticität bestehen. Was den ersten Punkt betrifft, so zeigt die Theorie, dass bei sonst gleichen Umständen der Radius der Druckfläche der Kubikwurzel aus dem Krümmungsradius der Kugelfläche, also die Druckfläche selbst seiner $2 / 3$. Potenz direct proportional ist. Bei gleichem Gesammtdruck ist also der Einheitşdruck und folglich auch der Maximaldruck der Kubikwurzel aus dem Krümmungsradius proportional. Ist nun dieser Maximaldruck die maassgebende Grösse, so muss sein der Elasticitätsgrenze entsprechender Grenzwerth unabhängig vom Krümmungsradius sein. Es muss also der Grenzwerth des Gesammtdruckes dem Quadrate des Grenzradius der Druckfläche proportional sein, oder, wenn man hierin mit Hülfe der obigen Beziehungen den Radius der Druckfläche durch Gesammtdruck und Krüm. mungsradius ausdrückt, es muss der Grenzwerth des Gesammtdruckes mit dem Quadrate des Krümmungsradius wachsen. Auf den zweiten Punkt soll hier zunächst nicht näher eingegangen werden, es sei nur bemerkt, dass unter sonst gleichen Umständen die Grösse der Druckfläche von einer Combination der Elasticitätsconstanten der Stoffe ab. bängt; in dem hier vorerst ausschliesslich zu betrachtenden Falle, dass die beiden Körper aus dem gleichen Stoffe bestehen, wird diese Abhängigkeit natürlich eine besonders einfache.

Um die angefuhrten Gesetze in Formeln zu bringen, sollen folgende Bezeichnungen eingefübrt werden. Es sei:

$\varrho$ der Krümmungsradius der KugelHäche in Millimetern;

$p$ der ausgeübte Druck in Kilogrammen;

$P$ sein Grenzwerth, d. h. sein Werth im Augenblicke des Eintrittes einer bleibenden Deformation;

$p_{1}$ der Einheitsäruck im Mittelpunkte der Druckfläche, also der Maximaldruck, in Kilogrammen pro Quadratmillimeter;

$P_{1}$ sein Grenzwerth, also die "theoretische Härte";

$d$ der Durchmesser der Druckfläche in Millimetern (da 
er in den Beobachtungen unmittelbar auftritt, ist er dem oben stets betrachteten Radius vorzuziehen);

$D$ sein Grenzwerth in Millimetern;

$H$ die wahre Härte, die, wie sich zeigen wird, von der theoretischen in einer gewissen Hinsicht verschieden ist;

$q$ zur Abkürzung der Quotient $p / d^{3}$;

$Q$ sein Grenzwerth;

$f$ die Grösse der Druckfläche in Quadratmillimetern;

$F$ ihr Grenzwerth;

$E$ der Elasticitätsmodul des Materiales in Kilogrammen pro Quadratmillimeter;

$\mu$ seine Elasticitätszahl, d. h. das Verbältniss der Quercontraction zur Längsdilatation;

$E^{\prime}$ zur Abkürzung der Quotient $E /\left(1-\mu^{2}\right)$.

Schliesslich sollen eckige Klammern bedeuten, dass die eingeschlossene Grösse nicht in obigen absoluten Maassen, sondern in den zufälligen Beobachtungsmaassen ausgedrückt ist.

Hiernach ergeben sich folgende Formeln:

$$
\begin{aligned}
f=\frac{\pi}{4} d^{2}, & F=\frac{\pi}{4} D^{2}, \\
p_{1}=\frac{3}{2} \frac{p}{f}=\frac{6}{\pi} \frac{p}{d^{2}}, & P_{1}=\frac{3}{2} \frac{P}{F}=\frac{6}{\pi} \frac{P}{D^{2}},
\end{aligned}
$$

für ein und dasselbe $\rho$ und $E^{\prime}$ :

$$
\begin{gathered}
\frac{d}{\sqrt[3]{p}}=\text { const., } \\
q=\text { also } \frac{d^{*}}{p}=\text { const., }
\end{gathered}
$$

ein constanter Wertb, mit dem also auch der Grenzwerth $Q$ übereinstimmt. Folglich für gleiches $\varrho$ und $E^{\prime}: p_{1} / \sqrt[3]{p}=$ const. Für verschiedene $\varrho$, aber ein und dasselbe $E^{\prime}: d / \sqrt[3]{p \underline{p}}=$ const., also:

$$
\varrho q=\text { const. }
$$

für verschiedene $g$ und $E^{\prime}$ :

$$
d=\sqrt[3]{\frac{12 p Q}{E^{\prime}}}, \quad D=\sqrt[3]{\frac{12 \overline{P Q}}{E^{\prime}}}
$$

für verschiedene $\underline{o}$, aber gleiches $E^{\prime}$ : 


$$
P_{1}=\text { const., also: }\left\{\begin{array}{l}
\frac{P}{D^{4}}=\text { const., } \\
\frac{P}{\varrho^{2}}=\text { const., } \\
\frac{D}{\rho}=\text { const. } ;
\end{array}\right\}
$$

drei Gleichungen, welche nur verschiedene Ausdrucksweisen einer und derselben Beziehung sind. Endlich für ein bestimmtes $\varrho$ und $E^{\prime}$ die theoretische Härte:

$$
P_{1}=\frac{6}{\pi} \underset{D^{2}}{P}=\frac{1}{\pi} \sqrt[3]{\frac{3}{2} \frac{E^{\prime 2} P}{\varrho^{2}}}=\frac{6}{\pi} \sqrt[3]{P q^{2}}
$$

und nebenbei die Elasticitätsconstante $E^{\prime}$ :

$$
E^{\prime}=12 \varrho q \text {. }
$$

§ 3. Methode.

Aus dem Vorstehenden ergibt sich, dass man, um aus den Erscheinungen bei der Berührung einer ebenen und einer kugelförmigen Fläche gleichen Materiales die Härte desselben ableiten zu können, den Druck, unter welchem die Berührung stattfindet, bis zur Elasticitätsgrenze steigern, den Augenblick, in welchem diese erreicht ist, genau feststellen und in diesem Augenblicke zwei Grössen: den Gesammtdruck und den Durchmesser der Druckfläche messen muss. Die erste der Formeln (4) gibt dann die theoretische Härte. Für die Genauigkeit des Ergebnisses ist es offenbar misslich, dass man jede der beiden Grössen $\boldsymbol{P}$ und $D$ nur einmal messen kann, und es erscheint wünschenswerth, wenigstens für die Grösse $D-$ denn für $P$ ist es augenscheinlich nicht möglich - hierin Wandel zu schaffen; dazu können die beiden anderen Formeln (4) Anwendung finden. Die eine von ihnen erfordert, ausser der Messung von $P$ noch die Kenntniss von $\varrho$ und $E^{\prime}$; während man nun $\varrho$ als aus der Herstellung der Kugelfläche bekannt annehmen kann, würde man gezwungen sein, $E^{\prime}$, also $E$ und $\mu$ entweder aus den für den betreffenden Stoff vorliegenden Messungen zu entlehnen oder diese Messung an anderen Stücken desselben Materiales selbst vorzunehmen, beides wenig empfehlenswerthe Auskunftsmittel, da die Elasticitätsverhältnisse bekanntlich mit der kleinsten Verschiedenheit des Stoffes und selbst von individuellem 
Stück zu Stück oft nicht unerheblich variiren. Dagegen ist die letzte der Formeln (4) in jeder dieser Hinsichten durchaus brauchbar; sie setzt nämlich ausser der Messung von $\boldsymbol{P}$ lediglich die Kenntniss von $q$ voraus, die man sich, da bei zunehmendem Gesammtdruck $q$ constant bleibt, aus einer grösseren Zahl von Messungen bei wachsendem $p$ verschaffen kann, für die man also nicht auf die Messung des einzigen Grenzwerthes beschränkt ist. Im Gegentheil, es kann sogar nur vortheilhaft sein, diesen Grenzwerth $Q$, falls er von den ubrigen Werthen abweichen sollte, nicht mit zur Bildung des Mittelwerthes von $q$ zu benutzen, da in dem Augenblicke, wo man ibn beobachten kann, der Körper schon eine bleibende Deformation erfahren hat. Dass man auf diese Weise gezwungen wird, die Drucksteigerung nach und nach vorzunehmen, kommt nicht in Betracht, da ein solches Verfahren ohnehin geboten ist, wenn man nicht aus diesem oder jenem leicht ersichtlichen Grunde für den Grenzdruck einen zu grossen oder zu kleinen Werth zu finden, Gefahr laufen will.

Am einfachsten ist der Eintritt der Elasticitätsgrenze, wie schon erwähnt, bei spröden Körpern zu constatiren, und zwar durch das plötzliche Auftreten eines Sprunges; nur in seltenen Fällen bereitet dieselbe sich durch allmählige Ausbildung einer Rille vor, und in diesen Fällen handelt es sich meist um Unregelmässigkeiten im Material oder um Abweichungen von dem Charakter der Sprödigkeit. Es wurden daher zunächst spröde Körper ins Auge gefasst und die Methode vorerst dem Umstande angepasst, duss fast alle spröden Körper, namentlich die. Gläser und die meisten Krystalle, mehr oder weniger durchsichtig sind.

Der Körper mit kugelförmiger Fläche wird in Form einer planconcaven Linse von 1 bis $30 \mathrm{~mm}$ Krummungsradius, der Körper mit ebener Fläche in Form einer planparallelen Platte von rund $11,6 \mathrm{~mm}$ freiem Durchmesser und $8 \mathrm{~mm}$ Dicke angewendet, also von einer im Vergleich zur Breite hinreichenden Dicke, um eine Durchbiegung der Platte als solcher auszuschliessen. Die Platte ist fest, die Linse frei aufgestellt; der Druck wird, durch Vermittelung eines Hebels, durch Gewichte erzeugt. Die Druckfläche und das Auftreten des Sprunges werden, um naheliegenden Einwän- 
den za begegnen, in unveränderter Drucklage von Platte und Linse beobachtet, und zwar, da es sich um sebr kleine Grössen handelt, mit einem Mikroskop, normal durch die Platte hindurch. Die Druckfläche erscheint dabei als ein dunkler, kreisförmiger Fleck, der ebenso wie die ihn umgebenden Ringe als Interferenzerscheinung aufzufassen ist; tubrigens finden auch die Durchmesser dieser Ringe, wie sich zeigen wird, bei den Beobachtungen Verwerthung. Die Einzelheiten der Methode müssen an der Hand des Apparates besprochen werden.

\section{§ 4. A p parat.}

Der durch freundliche Vermittelung von Hrn. Abbe in dem hiesigen optischen Institute von $\mathrm{Z}$ ei s 8 ausgefuhrte Apparat, bei dessen Construction meine hiesigen Fachgenossen, insbesondere aber Hr. Abbe selbst, mir mit werthrollen Rathschlägen zur Hand gingen, ist in der Figur in einem schematischen Schnitt mit Fortlassung nebensächlicher Dinge dargestellt. Er ist so gebaut, dass er genügende Festigkeit besitzt, um den grossen Drucken, denen er auszusetzen ist, gewachsen zu sein; zugleich ist or zur möglichsten Vermeidung von Erschütterungen an einem Pfeiler eines Kellerzimmers mittelst starker Bänder montirt. Die gusseiserne Grundplatte $G G^{\prime}$ hat T förmigen Querschnitt, $730 \mathrm{~mm}$ Länge, $75 \mathrm{~mm}$ Breite und der Länge nach einen centralen Schlitz. Der auf ihr aufgeschraubte Träger $T$ enthält die Lager $t$ für die Schneiden $D$, welche den Drehpunkt für den schmiedeeisernen zweiarmigen Hebel $H H^{\prime}$ bilden. Der kürzere linke Schenkel $H$ hat in $50 \mathrm{~mm}$ horizontalem Abstande von der Drehaxe eine ringförmige Erweiterung ${ }^{\prime}$ ', deren conische Hohlung zur Aufnahme des die Linse $L$ tragenden Zapfens $Z$ dient. Von dem etwa zehnmal so langen rechten Hebel$\operatorname{arm} \boldsymbol{H}^{\prime}$, der in der Schneide $c$ endet, ist in der Figur ein längeres Stück weggelassen. Die Platte $p$ aus dem zu untersuchenden Material befindet sich in der centralen Durchbohrung der oberen Platte oo' eines Trägers, welcher aus dieser, der unteren Platte $u$ und einem Paar sie verbindender starker Säulen besteht, von denen in der Figur, da sie mit dem Linsenzapfen in einer Linie liegen, nur ein Stumpf $s$ ange- 
deutet ist. Die Platte o o' ist $16 \mathrm{~mm}$ stark, mit ihrer unteren Fläche schliesst diejenige der zu untersuchenden Platte $p$ in einer Linie ab. Der ganze Kasten lässt sich in dem Schlitz der Grundplatte verschieben und mittelst der kräftigen Fliugelschraube $S_{1}$ derart feststellen, dass ein gewünschter Punkt der Platte $p$ genau über den höchsten Punkt der Linse $L$ kommt. In ähnlicher Weise lässt sich das Mikroskop $M$ verschieben und mittelst der Flügelschraube $S_{3}$, sowie auf Grund der Form des Statives ebenfalls genau vertical über dem höchsten Punkte der Linse aufstellen. Die Linse $L$ ist in den $Z$ apfen $Z$ eingekittet, die Platte $p$ in die Platte oo', in die sie genau passt, einfach eingesetzt, am Herausfallen wird sie durch zwei uber ihren Rand geschobene Scharniere verhindert. Das Mikroskop enthält ein Ocularmikrometer $m$ und empfängt seine Beleuchtung, da sie von unten wegen Behinderung durch Platte und Linse nicht möglich ist, von der Seite, und zwar durch eine Gasflamme $F$, deren Strahlen durch eine Oeffnung im Rohr auf ein rechtwinkeliges, nur dessen eine Hälfte einnehmendes Prisma $n$ fallen, von hier nach unten und von dort wieder nach oben reflectirt werden, um endlich in der freien Hälfte des Rohres zum Auge des Beobachters zu gelangen. Der rechte Hebelarm $H^{\prime}$ kann mittelst der Schraube $A$ in so hoher, und folglich der kurze Hebelarm $H$ in so tiefer Stellung festgelegt werden, dass eine vorzeitige Berührung zwischen Linse und Platte ausgeschlossen ist. Wird $A$ herabgelassen, so würde der lange Hebelarm bei weitem das Uebergewicht gewinnen, wenn dieses nicht durch den schmiedeeisernen Arm $W$ mit dem Laufgewicht $w$ ausgeglichen werden könnte; die Form von $W$ war durch die Umstände geboten, ihre nachtheilige Wirkung auf die Empfindlichkeit des Hebels ist aber durch Hochlegung des letzten Armgliedes mit dem Laufgewicht $w$ wieder aufgehoben. Letzteres wird so gestellt, dass zwischen Linse und Platte noch ein kleiner Zwischenraum bleibt, und alsdann durch Aufsetzen kleiner Ringe $r$ auf den Stift $q$ die Berührung eben hergestellt, was sich an der Verwandlung des bunten Interferenzcentrums in ein schwarzes zu erkennen gibt. Zur Wiederherstellung dieses Zustandes in jedem gewünschten Augenblicke und gleichzeitig zur Präcisirung des 
Verlaufes der Versuche dient der am Ende der Grundplatte mittelst des Trägers $U$ aufgeschraubte Hebel $K K^{\prime}$. Das Gehänge $R$, an dessen Haken verschieden grosse Waagschalen angehăngt werden können, hat nämlich die Form eines Rahmens, in dessen Innerem, in gewissem Abstande übereinander, das Lager $e$ und die Schneide $c^{\prime}$ angebracht sind; wird nun durch Senkung der Schraube $B$ der Hebelarm $K$ gesenkt, also $K^{\prime}$ gehoben, so nimmt das an diesem Hebel angebrachte Lager $e^{\prime}$ die Schneide $c^{\prime}$ des Gehänges auf, hebt dieses somit in die Höhe und entlastet dadurch den Haupthebel $\boldsymbol{H H}^{\prime}$; wird umgekehrt $B$ hochgeschraubt, so senkt sich das Gehänge, die Schneide $c$ nimmt $e$ auf, und der Haupthebel wird belastet. Durch alle diese Einrichtungen wird es erreicht, dass im Laufe einer ganzen Versuchsreihe alle Stösse und plötzlichen Aenderungen ausgeschlossen bleiben, und es erfolgt die Belastung durch hinzugefügte Gewichte, durch Drehen der Schraube $B$ und unter Mitwirkung der Federung des Hebelarms, in beliebig langsamer und allmähliger Weise. Im allgemeinen wurde hiernach vor jeder Steigerung des Druckes durch vollständige Entlastung die drucklose Berührung zwischen Linse und Platte wiederhergestellt (siehe jedoch weiter unten).

85. Aligemeines über die Beobachtungen.

Der Gang einer normalen Versuchsreihe - vorbehaltlich späterhin eintretetender Vereinfachungen - gestaltet sich folgendermaassen. Man reinigt Linse und Platte sorgfältig von Staub, Fettigkeit, Wasser u. s. w. und bringt alsdann jene in den Zapfenträger, diese in den Plattenkasten, in welchen man sie so fest wie möglich festschraubt. Man stellt die Schraube $A$ so hoch, dass man den Plattenkasten ohne Gefahr der Berührung der Linsenkuppe über diese bringen kann, schiebt das Mikroskop daruber und sucht eine geeignete und den besonderen $Z_{\text {wecken }}$ der Versuchsreihe entsprechende Plattenstelle für die Berührung aus. In dieser Stellung zieht man $S_{1}$ fest an, alsdann, nachdem man durch Verschiebung des Mikroskops die Druckstelle möglichst in die Mitte des Gesichtsfeldes gebracht hat, auch $S_{2}$. Die Schraube $A$ hat man hierbei, da man sonst die Druckstelle 
nicht sieht, schon mehrfach, aber am besten nur bis nahe zur Herstellung der Berührung, lüften müssen; jetzt lüftet man sie, bis die Berührung eben erfolgt; ist dies der Fall, ehe der Hebel frei schwebt, so ist das ein Zeichen dafür, dass die lange Seite zu schwer ist, und man muss $w$ weiter hinaus schieben, bis schliesslich, eventuell unter Auflage eines kleinen Ringes auf $q$, bei freiem Hebel eben Berührung eintritt; man kann hier bis auf $1 \mathrm{~g}$ Genauigkeit gelangen viel mebr, als später von Bedeutung sein wird. Nun beginnt man mit der Belastung und benutzt als erste solche das Gehänge $R$, indem man die bisher möglichst tief stehende Schraube $B$ hebt, bis zwischen $e$ und $c$ Berührung eintritt, und dann ganz langsam noch weiter, bis die Uebertragung der Last $R$ von $K K^{\prime}$ auf $H H^{\prime}$, die infolge der Federung allmählich erfolgt, beendet ist; man kann dabei $B$ so langsam drehen, dass das im Mikroskop beobachtete Wachsthum des Fleckes ein kaum merkliches ist, der Substanz also gestattet ist, ganz langsam in den $Z$ wangszustand überzugehen. Fürchtet man, dass die $227 \mathrm{~g}$, welche das Gehänge wiegt, als erste Etappe schon zu viel seien, so hängt man kleinere Gewichte mittelst einer Schnur an. Hat man den Fleckdurchmesser gemessen, so befreit man den Haupthebel wieder rom Gebänge, um nun die Waagschale an dasselbe anzuhängen, und, nach erfolgter Belastung des Haupthebels, wie$\operatorname{der} d$ zu messen. So setzt man dies fort, fügt schliesslich, wenn man Grund zu der Annahme hat, dass man der Grenze nahe sei, nur noch kleine Gewichte hinzu, und erhält schliesslich, wenn der Sprung eingetreten ist, die Grössen $\boldsymbol{P}$ und $\boldsymbol{D}$. Diese Methode hat den Vortheil, dass man in Ruhe und ohne weitere Vorsicht Gewichte auflegen kann, ohne dass diese Manipulationen die Druckstelle beeinflussen; sie hat freilich das Eigenthümliche, dass man, ehe man zu den Endwerthen gelangt, Platte und Linse mehrfach den Cyclus: Zwangszustand - natürlicher Zustand, lin und her durchlaufen lässt. Es wurden daher auch einige Reihen mit stetig wachsender, nie unterbrochener Belastung ausgeführt, dabei aber die Gewichte ganz sacht aufgesetzt, anfangs noch gestützt und ihre Last erst nach und nach auf die Waagschale übertragen; in der Nähe der Grenze wurde überdies Sand, 
welcher ganz behutsam aufgeschüttet wurde, als Zusatzgewicht benutzt. Dabei zeigte sich nun, dass zwar eine Reihe von Einzelheiten anders ausfiel, dass Endziel der vorliegenden Untersuchung aber nicht alterirt wurde. Ganz unzulässig ist es dagegen, und zwar auch schon im Anfang der Reihe, d. h. bei kleinen Drucken, die Belastung rasch herzustellen, d. h. die Schraube $B$ rasch zu drehen; der Sprung tritt dann, erklärlich genug, stets zu früb ein, und zuweilen um ein beträchtliches.

Ausser den zusammengehörigen Werthen von $p$ und $d$, $P$ und $D$, wurden meist noch einige andere Grössen beobachtet, theils zu Correctionszwecken (s. w. u.), theils für spätere Betrachtungen. So namentlich die Durchmesser der Newton'schen Ringe und der Durchmesser des Sprunges. In Bezug auf letzteren sei hier, indem die weitere Untersuchung einer besonderen Mittheilung vorbehalten wird, nur angeführt, dass er - bei isotropen Stoffen - in der That kreisförmig, mit der Druckfiäche concentrisch, aber mit ihrem Rande nicht zusammenfallend ist, sondern diesen in einem bestimmten Gesetzmässigkeiten unterworfenen Abstande umgibt, während er bei Krystallen eine Gestalt hat, welche zwischen einem Kreise und einem Polygon (Sechseck, Rhombus, Dreieck u. s. w.) die Mitte bält.

Hinsichtlich der Genauigkeit, mit der sich die Messungen ausfuhren lassen, ist nur in Bezug auf den Durchmesser des Flecks etwas hinzuzufügen. Es zeigte sich, dass der Fleck am deutlichsten wurde bei schwacher Beleuchtung, weil dann die Flamme klein und die störende Reflexion von der oberen Plattenfläche gering ausfiel. Im übrigen ist die Schärfe des Fleckrandes in verschiedenen Fällen und selbst bei demselben Fleck zuweilen an verschiedenen Stellen eine sehr verschiedene, aber meist ausreichend, um 0,1 Scalentheil noch gut schätzen zu können.

Schliesslich sei zur öconomischen Seite der Versuche noch bemerkt, dass man mit jeder Linse und jeder Platte zahlreiche Messungen, wio solche behufs Gewinnung ron Mittelwerthen nothwendig sind, ausführen kann, mit jener, weil sie (s. w. u.) meist unversehrt bleibt, mit dieser, weil sie Raum für 30-40 Sprünge auf jeder Seite darbiotet; zur 
Festlegung der einzelnen Versuche ist es dabei gut, die augenblicklich nicht benutzte Seite in unschädlicher Weise mit einem Coordinatennetz zu versehen. Es ist überraschend, wie wenig sich die Sprunge gegenseitig beeinflussen, selbst wenn sie sehr nahe bei einander liegen; ja, es wurden sogar zuweilen bei sich umschliessenden oder schneidenden Sprüngen ganz brauchbare (aber natürlich weiterhin nicht benutzte) Werthe erhalten. Eine Anschauung ron diesen Verhältnissen gibt die den freien Theil einer Platte darstellende Fig. 2; obwohl ihr bereits 28, je nach der erzeugenden Linse verschieden grosse Sprunge beigebracht sind, bietet sie doch, wie man sieht, noch Raum für weitere Versuche.

\section{\$ 6. Constanten und Fehlerquellen.}

Die zunächst zu ermittelnden Constanten des Apparats sind der Werth eines Theiles des Ocularmikrometers und das Verhältniss der Hebelarme. Jener wurde durch Vergleichung mit einem Objectmaassstab ermittelt und zwar am genauesten schliesslich in der Weise, dass eine Glasplatte, in deren Unterseite Zehntelmillimeter eingeritzt waren, in der Lage $P$ beobachtet wurde. Es fand sich:

$$
27 \mathrm{sc}=1 \mathrm{~mm} \text {, }
$$

mit einer gleich zu besprechenden Abweichung am Rande. Das Verhältniss der Hebelarme hätte bei der Gestalt des Hebels auf directem Wege nur ungenau ermittelt werden können; es wurde daher an die Stelle des Linsenzapfens $Z$ ein Zapfen mit einer Spitze gebracht, auf diese eine kräftige Wagschale, die unter die Grundplatte herunterhing, gebracht und mit den aufgelegten Gewichten ins Gleichgewicht mit einer kleineren an das Gehänge $\boldsymbol{R}$ gehängten Wagschale nebst Gewichten gebracht. Es ergab sich auf diese Weise die Verhältnisszahl: $\quad v=\mathbf{9 , 8}$

mit einem wahrscheinlichen Fehler von $\pm 0,01$, d. $h$. etwa $1 / 1000$ des Werthes. Hiernach sind alle im Mikroskop gemessenen Längen mit $27 \mathrm{zu}$ dividiren, und aus dem beobachteten Gewicht, in dem natürlich das Gewicht von Gehänge und $W$ agschale mit enthalten ist, ergibt sich der wirkliche Druck zwischen Linse und Platte durch Multiplication mit 9,8.

Die dritte Constante ist die für verschiedene Reihen Ann. d. Phys, u. Chem. A. F. XLIII. 
verschiedene Linsenkrümmung. Eigene Messungen wurden hier nicht ausgeführt; vielnehr begnügte man sich, ausser den Werthen der Radien selbst auch die vermuthlichen Fehlergrenzen an der die Herstellung der Linsen leitenden Stelle zu ermitteln, und diese Fehlergrenzen gingen nie uber das Maximum von $0,1 \mathrm{~mm}$ hinaus, waren aber bei den stärker gewölbten Linsen noch beträchtlich kleiner.

Eine wichtige Frage ist ferner die, ob der im Mikroskop beobachtete schwarze Fleck seiner Grösse nach die Druckfläche unmittelbar wiedergibt, oder ob, und eventuell welche Correctionen vorerst noch vorzunehmen sind. Es sind hier vier Correctionen denkbar:

1) Wegen des Umstandes, dass eine fast rollständige Auslöschung des Lichtes bekanntlich nicht nur in der Berührungefläche ron Platte und Linse, sondern noch darüber hinaus bis zu der Stelle stattfindet, wo der verticale Abstand beider etwa $1 / 6$ Wellenlänge (je nach der Beleuchtung subjectiv etwas mehr oder weniger) beträgt, der Fleck also um so viel grösser erscheint, als die wirkliche Druckfläche. Man könnte diese Correction aus der durch die Drucktheorie gegebenen Gestalt der Linse in allgemeiner und auf jeden einzelnen Fall anwendbarer Weise berechnen, und es ist das in der That geschehen; einfacher und von gewissen, hier nicht năher zu erörternden Annahmen in geringerem Grade abhängig ist aber das Verfahren, welches auf der Erwägung beruht, dass der erste, den schwarzen Fleck umgebende dunkle Ring sich da befindet, wo der verticale Abstand von Linse und Platte eine halbe Wellenlänge beträgt. Nun kann man, da die Druckfläche relativ sehr klein ist, die Krümmung vernachlässigen und erbält also das Ergebniss, dass die abzuziehende Correction $1 / 3$ des Abstandes des ersten Ringes vom Fleckrande, wohlverstanden vom wahren Fleckrande beträgt; von dem der Beobachtung zugänglichen Abstande des Ringes rom scheinbaren Fleckrande beträgt sie hiernach die Hälfte von $\varepsilon$, und da diese Correction zu beiden Seiten des Durchmessers stattzufinden hat, muss man von $d$ gerade $\varepsilon$ abziehen. Hiernach, sowie unter Berücksichtigung einiger weiterer Umstände, kann man sich für einen bestimm. ten Stoff eine Tabelle der Correctionen als Functionen von 
Druck und Linsenkrümmung herstellen; man kann sich aber auch eine für alle Stoffe näherungsweise geltende Tabelle verschaffen, wenn man als Variable Iinsenkrümmung und Fleckgrösse einführt; diese Tabelle (die Fleckgrössen in Scalentheilen, die Correctionen in Zehntel Scalentheilen) sei hier wiedergegeben:

\begin{tabular}{r||c|c|c|c|c|c|c|c}
\hline \hline & $\rho=1$ & 3 & 4 & 5 & 10 & 12 & 15 & 30 \\
\hline \hline$d=$ & & & & & & & & \\
5 & 1 & 3 & 4 & 4 & 6 & 7 & 8 & 12 \\
10 & & 2 & 2 & 3 & 4 & 5 & 6 & 10 \\
15 & & 1 & 1 & 2 & 8 & 4 & 6 & 9 \\
20 & & & 1 & 2 & 3 & 3 & 5 & 8 \\
25 & & & & 1 & 2 & 3 & 4 & 7 \\
311 & & & & 1 & 2 & 2 & 3 & 6 \\
35 & & & & & 1 & 2 & 3 & 5
\end{tabular}

2) Wegen der astigmatischen Verzerrung des durch die Planplatte beobachteten Bildes. Es genüge hier die Bemerkung, dass bei der Kleinheit des Gesichtswinkels der betreffende Fehler über $1 / 1000$ des Werthes nicht hinausgeht, ausser wenn, was nie geschehen ist, an der äussersten Peripherie des Gesichtsfeldes beobachtet wird.

3) Wegen des Umstandes, dass der Fleck nicht die gekrümmte Druckfläche selbst, sondern ihre ebene Projection wiedergibt; auch dieser Fehler ist verschwindend klein, da selbst bei den kleinsten zur Anwendung gelangten Linsen und den stärksten Drucken der Grössenunterschied beider Flächen unter $2 / 1000$ bleibt.

4) Wegen der etwaigen Wirkung der durch den Druck deformirten Platte als Planconcavlinse. Eine solche Wirkung würde stattfinden, wenn das beobachtete Object um eine in Betracht kommende Strecke hinter der Concavfläche läge, was denkbar wäre, da dieses Object, d. h. die Peripherie des Flecks, nach dem Obigen an einer Stelle liegt, wo Platte und Linse sich nicht mehr berühren, und da keine Angaben darüber vorliegen, wo eigentlich in einem Falle wie der hier behandelte, die Interferenzerscheinung ihren Sitz hat. Durch verschiedene Beobachtungen, sowie durch eine Grenzrechnung, stellte sich aber heraus, dass diese Fehlerquelle die vorerwähnten an Einfluss nicht oder doch nicht wesentlich übertrifft. 
\$ 7. Prüfung der Theorie.

Als Material für die Versuche dienten drei von der hiesigen Glasschmelze Schott und Gen. herrührende Glas. sorten, von denen Sorte I als ziemlich weich, II als von mittlerer Hărte, III als ziemlich hart bezeichnet wurde; gerade in Anbetracht des Umstandes, dass es sich hierbei um drei Sorten eines und desselben Materials handelte, also nur verhältnissmässig geringe Härteunterschiede zu erwarten waren, mussten diese Versuche zugleich auch über die Em. pfindlichkeit der Methode entscheiden. Als viertes Material diente senkrecht zur Axe geschnittener Bergkrystall (Quarz), und zwar ebenso, wie bei den Glassorten in der Weise, dass Linse und Platte von einem einzigen Stück des Materials herrühren. Auf optischem Wege überzeugte man sich davon, dass die Abweichung der schliesslich benutzten Gläser von der Isotropie, sowie die Abweichung der Quarzplatten von der gewünschten Richtung nur eine sehr geringfügige war; wäre es nicht der Fall gewesen, so hätte sich das übrigens auch in der Lage und Gestalt der Sprünge offenbaren müssen, wie dies bei einigen, eigens zu diesem $Z$ wecke angestellten, hier aber nicht näher zu besprechenden Versuchen in der That der Fall war. Noch muss eine Frage beantwortet werden, die dem Leser sich unwillkürlich aufgedrängt haben wird, die Frage, ob und in wie weit es denn erlaubt sei, ein krystallisches Material zu wählen, während doch die Theorie nur für isotrope Körper gilt. Letzteres ist richtig; jedoch ist zu beachten, dass z. B. in der ersten der Formeln (4) lediglich der Zahlenfactor unrichtig sein könnte; erweisen sich die Beziehungen (1) und (2) empirisch als for die betreffenden Krystallkörper erfullt, so wird auch in der letzten Formel (4) nur der Zahlenfactor zweifelhaft sein können, und auch diese Ungewissheit wird man durch Prüfung der Beziehungen (3) und der zweiten Formel (4), sowie unter Zuhülfenahme bekannter Werthe von $E^{\prime}$ uf ein sehr geringes Maass zurückführen können, zumal schon aus der Bedeutung des Zahlenfactors sich ergibt, dass er in enge Grenzen eingeschlossen ist. Dazu kommt folgende Erwägung. Hertz hat seine Theorie nicht blos für den Fall kreisförmiger Druckflächen, sondern auch für denjenigen elliptischer Druck- 
flächen entworfen, und auch in diesem allgemeinen Falle wird der in Rede stehende Zahlenfactor 3/2. Hat also die geometrische Verschiedenheit der Richtungen auf ihn keinen Einfluss, so wird man annehmen dürfen, dass auch die elastische Asymmetrie ihn nicht haben wird - eine Betrachtung, die sich freilich nur auf die beiden in die Plattenebene fallenden Richtungen, nicht aber auch auf die Tiefendimension erstreckt. Immerhin muss zugegeben werden, dass die Genauigkeit der Resultate für Krystalle vielleicht nicht ganz so gross sein wird, wie für isotrope Stoffe. In qualitativer Hinsicht zeigt sich der Gegensatz zwischen isotropen Stoffen und Krystallen in sehr auffälliger Weise; während nämlich bei ersteren Druckfläche wie Sprung kreisformig sind, ist beim Bergkrystall zwar die Druckfläche ebenfalls ein Kreis, der Sprung aber hat eine Form, welche zwischen derjenigen eines Kreises und der eines regulären Sechsecks liegt eine Erscheinung, deren Verfolgung einer späteren Mittheilung vorbehalten bleiben muss. Als Krümmungsradien wurden in den verschiedenen Fällen solche von 1, 3, 4, 5, 10, $12,15,30 \mathrm{~mm}$ benutzt, der erst- und der letztgenannte Werth jedoch nur zu gewissen Versuchen (s. w. u.), da zu kleine Linsen wegen der Kleinheit der zu messenden Erscheinung zu grosse deshalb sich als ungünstig erweisen, weil alsdann die exact punktförmige Berührung zwischen Platte und Linse nicht stets mit Sicherheit hergestellt werden kann, die Belastung über die dem Apparat zuträgliche Grenze hinaus gesteigert werden muss und bei der Grösse der Sprünge eine Platte nur für wenige Versuche Raum bietet.

Die ersten Messungen mussten den $Z$ weck haben, die Theorie $\mathrm{zu}$ prüfen, also im wesentlichen $z \mathrm{u}$ untersuchen, $\mathrm{ob}$ und in wie weit die obigen Formeln (1), (2) und (3) Bestatigung finden. Erst dann konnte dazu ubergegangen werden, mittelst der Formeln (4) und (5) die Grössen $P_{1}$ und $E^{\prime}$ zu messen. ${ }^{1}$ )

1) In Bezug auf sämmtliche folgenden Zahlenangaben ist zu bemerken, dass dieselbeu zum Theil von den in den Göttiuger Nachrichten mitgetleilten um kleine Beträge abweichen; es rührt das theils von experimentellen Ergănzungen, theils von rechnerischen Correctionen her, die inzwischen eintreten zu lassen sich Gelegenbeit fand. 
1) Zur Prüfung der Formel (1) $q=$ const, also $p / d^{s}=$ const. wurden mit jedem Material zahlreiche Versuchsreihen unter wachsender Belastung ausgeführt. Hier genüge die Angabe einiger, weder besonders günstig, noch besonders ungünstig ausgewählter Beispiele.

Glas II. $\varrho=10$

\begin{tabular}{c|c|c}
\hline \hline$p]$ & {$[d]$} & $1000[q]$ \\
\hline \hline 227 & 8,9 & 321 \\
354 & 10,5 & 306 \\
554 & 12,1 & 313 \\
754 & 13,5 & 307 \\
954 & 14,6 & 306 \\
1354 & 16,4 & 307 \\
1554 & 17,1 & 311 \\
1677 & 18,0 & 288 \\
1925 & 18,7 & 294 \\
3177 & 22,1 & 294 \\
3225 & 22,2 & 295 \\
3725 & 23,4 & 291 \\
4547 & 24,6 & 306
\end{tabular}

Quarz. $\quad \varrho=12$.

\begin{tabular}{c|c|c}
\hline \hline$p]$ & {$[d]$} & $1000[q]$ \\
\hline \hline 754 & 12,4 & 396 \\
1254 & 15,0 & 371 \\
1677 & 17,0 & 342 \\
2677 & 19,6 & 356 \\
3177 & 20,5 & 369 \\
3677 & 21,6 & 368 \\
4390 & 23,0 & 359 \\
4800 & 23,7 & 361 \\
4887 & 23,9 & 357 \\
G 1as & III. & $0=4$. \\
854 & 10,0 & 854 \\
1154 & 11,0 & 866 \\
1754 & 12,6 & 877 \\
2454 & 14,3 & 876 \\
2479 & 14,4 & 866
\end{tabular}

Wie man sieht, ist $q$ in der dritten Reihe durchaus, in den beiden ersten Reihen in erster Annäherung constant; bei genauerem Zusehen zeigt sich, von den unregelmässigen Schwankungen abgesehen, bei den beiden ersten Reihen eine geringfügige Abnahme, welche sich z. B. darin ausspricht, dass in der einen Tabelle das Mittel der sieben ersten Zah. len 310, das der sechs letzten 295, in der anderen Tabelle das Mittel der fünf ersten Zahlen 367, das der vier letzten 361 ist - eine Abnahme, welche, nach Formel (5) auf eine schwache Abnahme von $E^{\prime}$, also auf eine Abnahme entweder des Elasticitätsmoduls $E$ oder der Elasticitätszahl $\mu$ mit wachsendem Drucke hinweist, also durchaus plausibel ist. Da sie meist sehr unbeträchtlich ist, in vielen Reihen aber sogar überhaupt nicht auftritt, soll sie hier nicht weiter verfolgt, sondern, der Theorie entsprechend, $q$ als constant betrachtet werden. Fasst man demgemäss sämmtliche Schwankungen von $q$ in einer Reihe als unregelmässige auf und berechnet hiernach Mittelwerth und wahrscheinlichen Fehler, so findet man in den drei obigen Beispielen: 


$$
\begin{gathered}
{[q]=0,3028 \pm 0,0016, \quad[q]=0,3643 \pm 0,0031 ;} \\
{[q]=0,868 \pm 0,003,}
\end{gathered}
$$

der wahrscheinliche Fehler beträgt also in den auf Glas bezüglichen Reihen nur etwa $1 / 2$ Proc., in der auf Quarz bezthglichen immer noch weniger als 1 Proc.

Um diesen Fehler noch weiter zu verringern, wurde jede Versuchsreihe unter Veränderung der Druckstelle mehrmals wiederholt. Die hierbei für verschiedene Plattenstellen oder für verschiedene Linsen von gleichem Material und Krüm. mungsradius sich ergebenden Werthe von $q$ weichen nun zwar zum Theil starker von einander ab, als nach obigem zu erwarten wäre, und es ist daher zu schliessen, dass diese Stellen wirklich etwas verschiedene Elasticităt besitzen; die Differenzen und die wahrscheinlichen Fehler der Hauptmittel sind jedoch, wie man sieht, uberraschend klein.

a) Glas I.

\begin{tabular}{c|c||c|c||c|c}
\hline \hline$\rho=1$ & \pm & $\rho=4$ & \pm & $\rho=12$ & \pm \\
\hline \hline 468 & - & 122,1 & 2,7 & 39,4 & 0,2 \\
429 & $=$ & 110,6 & 1,1 & 38,3 & 0,3 \\
502 & $=$ & 115,7 & 1,3 & 38,7 & 0,3 \\
454 & $=$ & 117,7 & 2,6 & 40,0 & 0,1 \\
429 & $=$ & $=$ & $=$ & $=$ & $=$ \\
442 & $=$ & $=$ & $=$ & $=$ & $=$ \\
505 & $=$ & $=$ & $=$ & $=$ & $=$ \\
448 & - & $=$ & $=$ & $=$ & $=$ \\
455 & $=$ & $=$ & $=$ & $=$ & $=$ \\
455 & $=$ & $=$ & $=$ & $=$ & $=$ \\
476 & $=$ & $=$ \\
456 & - & $=$ & $=$ & $=$ & $=$ \\
482 & $=$ & - & $=$ & - & - \\
449 & $=$ & - & - & 39,1 & 0,25 \\
476 & - & 116,5 & 1,6 & 39, &
\end{tabular}

b) Glas II.

\begin{tabular}{c|c||c|c||c|c||c|c}
\hline$\varrho=3$ & \pm & $\varrho=5$ & \pm & $\varrho=10$ & \pm & $\varrho=15$ & \pm \\
\hline 202,6 & 1,9 & 118,9 & 1,0 & 58,5 & 1,3 & 36,7 & 0,9 \\
196,3 & 3,4 & 111,6 & 3,0 & 57,2 & 1,3 & 37,3 & 0,4 \\
190,6 & 2,8 & 112,2 & 3,1 & 58,5 & - & 39,0 & 1,6 \\
195,7 & 4,7 & 115,0 & $\mathbf{2 , 6}$ & 59,7 & 1,5 & 39,2 & 0,7 \\
196,3 & 1,9 & 111,3 & 1,5 & 59,3 & 0,9 & - & - \\
- & - & 116,0 & 2,4 & 59,7 & - & - & - \\
- & - & 112,7 & $\mathbf{3 , 8}$ & 56,6 & $\mathbf{2 , 2}$ & - & - \\
- & - & 117,1 & 0,7 & 55,3 & 1,1 & - & - \\
\hline 195,4 & 1,2 & 114,9 & 0,7 & 58,3 & 0,4 & 38,3 & 0,4
\end{tabular}


c) Glas III.

\begin{tabular}{c|c||c|c||c|c|}
\hline \hline$\rho=4$ & \pm & $\rho=12$ & \pm & $\rho=30$ & \pm \\
\hline \hline 160 & - & 52,2 & - & 22,0 & 0,1 \\
167 & $=$ & 53,1 & - & 21,7 & 0,0 \\
151 & $=$ & 52,8 & - & 21,7 & 0,1 \\
168 & $=$ & 53,1 & - & 21,6 & 0,1 \\
167 & - & 53,6 & - & 21,9 & 0,1 \\
162 & - & - & - & 22,2 & 0,1 \\
\hline 162,5 & 1,7 & 53,0 & 0,2 & 21,85 & 0,05
\end{tabular}

d) Quarz.

\begin{tabular}{|c|c|c|c|c|c|}
\hline$\varrho=1$ & \pm & $\rho=4$ & \pm & $\rho=12$ & \pm \\
\hline 823 & - & 218 & - & 70,5 & - \\
\hline 848 & - & 208 & - & 69,2 & - \\
\hline & & 215 & & 71,9 & - \\
\hline & - & 210 & - & 67,3 & - \\
\hline 854 & - & 210 & - & 70,7 & - \\
\hline & & 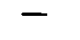 & & 71,5 & \\
\hline & & & & 70,9 & \\
\hline- & - & - & - & 69,8 & - \\
\hline & 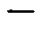 & - & - & 70,5 & - \\
\hline- & - & $\overline{--}$ & - & 72,0 & $n$ \\
\hline
\end{tabular}

3) Dass auch die Gl. (2) der Theorie erfüllt ist, also $\varrho q=$ const., zeigen folgende Angaben:

a) Glas I.

$\left.\begin{array}{rccc}\rho & 1 & 4 & 12 \\ q & 463 & 116,5 & 39,1 \\ \rho q & 463 & 466 & 469\end{array}\right\} \rho q=466 \pm 1$

b) Glas II.

$\left.\begin{array}{rcccc}\rho & 3 & 5 & 10 & 15 \\ q & 195,4 & 114,9 & 58,3 & 38,3 \\ \rho q & 586 & 575 & 583 & 575\end{array}\right\} \rho q=580 \pm 2$

c) Glas III.

$\left.\begin{array}{cccc}\rho & 4 & 12 & 30 \\ q & 162,5 & 53,0 & 21,8 \\ \rho q & 650 & 636 & 654\end{array}\right\} \rho q=647 \pm 4$

d) Quarz.

$\left.\begin{array}{cccc}\rho & 1 & 4 & 12 \\ q & 846 & 212,2 & 70,4 \\ \varrho q & 846 & 849 & 845\end{array}\right\} \varrho q=847 \pm 1$

3) Es bleibt nun noch übrig, die Gleichungen (3) zu prtifen. Was zunächst die für den Grenzdruck $\boldsymbol{P}$ selbst erhaltenen Zahlen betrifft, so schwankten diese in den verschiedenen Fällen zwischen 4 und $140 \mathrm{~kg}$, gingen also, zumal in Anbetracht der sehr kleinen Druckflächen, auf die sie sich vertheilten, bis zu unerwarteter Höhe hinauf. Bei den verschiedenen, unter gleichen Umständen, d. h. bei gleichem Material und gleicher Linsenkrümmung ausgefuhrten Reihen variirt $\boldsymbol{P}$ recht beträchtlich, wie folgendes, freilich $\mathrm{zu}$ den ungünstigeren gehörende Beispiel zeigt, wo der wahrscheinliche Fehler des Mittels $3^{1 / 2}$ Proc. beträgt. 


$$
\begin{aligned}
& \text { Glas III, } \rho=4 \text {. } \\
& \begin{array}{cccccccc}
P & 17,2 & 24,3 & 18,1 & 24,1 & 21,2 & 19,2 & 18,2 \\
D & 0,47 & 0,53 & 0,50 & 0,50 & 0,49 & 0,48 & 0,48
\end{array} \\
& \text { Mittelwerth von } P: \quad 20,3 \pm 0,7 \text {. }
\end{aligned}
$$

Allein es ist zu beachten, 1) dass dem grösseren $\boldsymbol{P}$ in allgemeinen auch das grössere $D$, also auch das kleinere $q$ entspricht, wodurch der grössere Theil der Schwankungen ausgeglichen wird, 2) dass in der Endformel $\boldsymbol{P}$ nur unter der Cubikwurzel vorkommt, alle Fehler sich also auf den dritten Theil reduciren.

Dagegen zeigt sich bei Anwendung verschiedener Linsenkrümmungen ein völlig unerwartetes Resultat. Die Gleichungen (3) sind nicht erfüllt, und $z$ war auch nicht näherungsweise; wohl aber lassen sich andere Beziehungen aufstellen, die sich mit grosser Genauigkeit als erfüllt erweisen. So ergab beispielsweise die Glassorte II:

\begin{tabular}{c||c|c|c|c}
\hline \hline$\rho$ & 3 & 5 & 10 & 15 \\
\hline$P: D^{2}$ & 81,7 & 67,0 & 56,6 & 49,8 \\
$P: \rho^{2}$ & 1,64 & 0,96 & 0,50 & 0,32 \\
$D: \rho$ & 0,142 & 0,119 & 0,094 & 0,080
\end{tabular}

Alle drei Verhältnisse nehmen also, statt constant zu sein, mit wachsendem $\rho$ ganz beträchtlich ab.

Das will sagen: Der Druck auf die Flächeneinheit, bei welchem ein Sprung in der Plutte eintritt, ist bei gleichem Material nicht unter allen Umständen derselbe, sondern er ist desto grösser, je gekrümmter die drückende Linse oder je kleiner die Druckfläche ist. Fine andere Formulirung jst die, dass der Gesammtdruck, bei welchem der Sprung eintritt, nicht dem Quadrat des Krümmungsradius proportional ist, eine dritte die, dass der Durchmesser der Druckfläche beim Eintritt des Sprunges nicht dem Krümmungsradius selbst proportional ist, sondern dass beide Grössen langsamer wachsen. Ein Blick auf die obige Tabelle zeigt nun sofort, dass die Zahlen der zweiten Horizontalreihe fallen, wie die $\varrho$ wachsen, und ähnlich einfache Wahrnehmungen lassen die anderen Reiben zu. Hiernach ergibt sich, dass die von der Theorie geforderten drei Be- 
ziehungen durch die folgenden der Beobachtung entsprechenden zu ersetzen sind:

1) $P$ proportional nicht mit $D^{2}$, sondern mit $D^{3 / s}$,

2) $P \quad " \quad " \quad " \varrho^{2}, \quad " \quad$,

3) Nicht $D$; sondern $D^{3}$ proportional mit $\varrho$. In wie weit dies der Fall ist, zeigt die folgende Zusammen. stellung.

\begin{tabular}{c||c|c|c|c||c}
\hline \hline$\varrho$ & 3 & 5 & 10 & 15 & Mittel \\
\hline$P: D^{8 / 2}$ & 53,4 & 52,0 & 54,8 & 54,5 & $53,7 \pm 0,4$ \\
$P: \varrho$ & 4,93 & 4,78 & 5,04 & 4,80 & $4,89 \pm 0,04$ \\
$D^{3 / 2}: \varrho$ & 0,092 & 0,092 & 0,092 & 0,088 & $0,091 \pm 0,001$
\end{tabular}

Die wahrscheinlichen Fehler betragen also sämmtlich nur 1 Proc.

Für die drei anderen Stoffe fanden sich die Beziehungen zwar ebenfalls erfült, jedoch, da hier zunächst nur je zwei Werthe von $\varrho$ (4 und $12 \mathrm{~mm}$ ) vorlagen, mit geringerer beweisender Kraft. Es wurde daher aus zweien dieser Stoffe, dem weichsten Glase (I) und dem Bergkrystall, noch je eine Linse mit einem dritten Radius hergestellt, für dessen Wahl einmal der $W$ unsch maassgebend war, dem Begriff der Spitze näher zu kommen, also einen recht kleinen Radius zu nehmen, andererseits die Erwägung, dass, wenn der Grenzwerth des Einheitsdruckes vom Linsenradius abhängt, derjenige Werth eine besondere absolute Bedeutung haben wird, welcher dem Werth $. \rho=1$ entspricht. Bei den Versuchen mit diesen stark gekrümmten Linsen traten nun zwar, wie zu erwarten war (s. oben), etwas grössere Unregelmässigkeiten als bei den anderen auf, das Gesammtergebniss aber ist und bleibt die volle Bestätigung der obigen Beziehung:

Glas I.

Theoretisch constante Verhältnisse.

\begin{tabular}{c||c|c|c}
\hline$\rho$ & 1 & 4 & 12 \\
\hline$P: D^{2}$ & 111,1 & 71,0 & 48,1 \\
$P: \varphi^{2}$ & 6,37 & 1,65 & 0,53 \\
$D: \rho$ & 0,240 & 0,153 & 0,103
\end{tabular}


Absolute Härtemessung.

In Wahrheit constante Verhältnisse.

\begin{tabular}{c||c|c|c|c}
\hline$\rho$ & 1 & 4 & 12 & Mittel \\
\hline$P: D^{3 / 2}$ & 54,4 & 55,4 & 53,5 & $54,4 \pm 0,3$ \\
$P: \rho$ & 6,37 & 6,59 & 6,35 & $6,44 \pm 0,5$ \\
$D^{2 / 2}: \varrho$ & 0,117 & 0,119 & 0,119 & $0,118 \pm 0,001$
\end{tabular}

Bergkrystall.

Theoretisch constante Verhältnisse.

\begin{tabular}{c||c|c|c}
\hline \hline$\rho$ & 1 & 4 & 12 \\
\hline$P: D^{2}$ & 149,8 & 95,2 & 66,9 \\
$P: \varrho^{2}$ & 5,05 & 1,31 & 0,42 \\
$D: \varrho$ & 0,183 & 0,118 & 0,079
\end{tabular}

In Wahrbeit constante Verhaltnisse.

\begin{tabular}{c||c|c|c||c}
\hline \hline$\rho$ & 1 & 4 & 12 & \multicolumn{1}{c}{ Mittel } \\
\hline \hline$P: D^{3 / 2}$ & 64,3 & 65,8 & 65,2 & $65,1 \quad \pm 0,3$ \\
$P: \rho$ & 5,05 & 5,22 & 5,05 & $5,11 \pm 0,04$ \\
$D^{3 / 2}: \rho$ & 0,0786 & 0,0793 & 0,0775 & $0,0785 \pm 0,0004$
\end{tabular}

Auch hier betragen die wahrscheinlichen Fehler nur $1 / 2$ bis 1 Proc.

Hiernach ergibt sich, dass auch die nach der letzten der Formeln (4) berechnete Hertz'sche Harte $P_{1}$ für verschiedene $\varrho$ verschieden ausfallt, dass man dagegen gleiche Zahlen erhält, wenn man jedes so gefundene $P_{1}$ entweder mit $\sqrt{D}$, oder, was einfacher ist, mit $\sqrt[3]{\varrho}$ multiplicirt.

Somit ist die Thatsache constatirt, dass die Erfahrung zwar in allen übrigen Punkten die Theorie mit überraschender Genauigkeit bestätigt, in dem letzten und wichtigsten Punkte aber von ihr ganz erheblich, und zwar nach bestimmtem Gesetze abweicht. Diese Thatsache fordert zu erneuter Betrachtung der Voraussetzungen jener Theorie auf.

1) Diese Theorie macht u. A. die Voraussetzung, dass die Druckfläche klein sei im Vergleich zur Kugelfäche, und es kann zweifelhaft erscheinen, ob diese Forderung der Theorie bei obigen Versuchen erfült ist. Nun steigt allerdings das Verhältniss $\boldsymbol{R}$ (Grenzradius der Druckfläche) : $\boldsymbol{\varphi}$ (Linsenradius) bis zum Werthe 1:11, also zu einem Verhält- 
nisse, welches an sich gewiss nicht mehr als sehr klein zu betrachten ist; aber schliesslich kommt es lediglich darauf an, ob dieses Verhältniss so gross ist, dass die in der Theorie darauf basirten Annahmen, betreffend Druckrichtung, Druckcomponenten, Krümmung und Grösse der Druckfläche, nicht mehr erfüllt sind. Das ist aber, wie zum Theil schon aus den obigen Andeutungen hervorgeht, und sich noch eingehender zeigen liesse, nicht der Fall; die Abweichungen liegen vielmehr innerhalb der Fehlergrenzen der Versuche oder fallen höchstens ein klein wenig aus ihnen heraus. Es zeigt sich dies auch in der Constanz von $q$ bei steigendem Druck, die gerade bei demjenigen der drei oben gegebenen Beispiele am schärfsten hervortritt, in welchem $\varrho$ am kleinsten ist.

2) Nach der Theorie kann es ferner - obwohl eine nähere Betrachtung hierfür Anhaltspunkte gibt (s. unten) befremden, dass der Sprung die Druckfläche in einem gewissen Abstande umgibt, und man könnte den Vorschlag machen, bei der Berechnung der obigen Verhältnisse statt der $D$ einmal die Sprungdurchmesser zu benutzen; das gefundene empirische Gesetz bleibt dann aber unverändert bestehen.

3) Dass es nicht die relativ zu beträchtliche Grösse der Druckfläche ist, welche die Abweichungen hervorbringt, ergibt sich am besten daraus, dass alsdann die $\mathrm{Abweichungen}$ desto kleiner werden müssten, je günstiger, $d$. h. je kleiner das Verbältniss $R: \varrho$ wird; das ist aber nicht der Fall, sie sind vielmehr einheitlich durch die obigen Beziehungen bestimmt; und z. B. für Glas III werden die Verhältnisse $P: D^{2}$ noch für $\varrho=4$ und $\varrho=12$ kolossal verschieden, nämlich 83,9 und 56,4 , obgleich hier das Verhältniss $R: \varrho$ nur noch $1: 16$, resp. $1: 23$ ist. Um es aber noch weiter zu verkleinern, wurde von diesem Glase eine sehr flache Linse von $\varrho=30 \mathrm{~mm}$ bergestellt; für sie fand sich $R: \varrho$ wie $1: 38$, und es müsste sich daher für $P: D^{2}$ ein mit dem für $\rho=12$ gefundenen übereinstimmender oder höchstens wenig von ihm abweichender Werth ergeben, wenn jener Standpunkt richtig wäre; es findet sich aber 39,6 gegen 56,4 , also ein wiederum 
ganz wesentlich kleinerer Werth, während das Verhältniss $P: D^{3 / 3}$ wieder denselben Werth annimmt.

4) Uebrigens ist zu beachten, dass die Werthe ron $P: D^{2}$ für verschiedene Stoffe in einem von $\rho$ ganz unabhängigen Verhältnisse zu einander stehen, nämlich für die untersuchten Stoffe in dem Verhältniss:

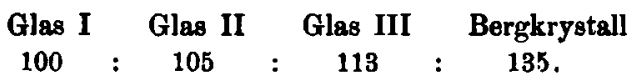

Die Gewinnung von relativen Härtezahlen ist also von den berührten Verhältnissen gänzlich unabhängig, und für die praktische Anwendung ist hiermit schon ein wesentlicher Fortschritt erzielt.

5) Wie es kommt, dass die absoluten Werthe der Härte eine so starke Abhängigkeit vom Krümmungsradius zeigen, dafür kann ich Beweisendes vorläufig nicht anführen, und ich gebe zu, dass dies eine principielle Lücke in der Lösung des Problemes lässt. Ich möchte aber zwei auf die Ausfulllung derselben bezügliche Vermuthungen nicht unerwähnt lassen. Die eine derselben ist folgende. Infolge der experimentell gefundenen Beziehungen liefert die letzte der Formeln (4) bei Benutzung verschiedener Linsen sehr verschiedene Werthe für $P_{1}$, also, wenn $P_{1}$ die Härte ist, für die Harte der betreffenden Platte. Da dies keinen Sinn hat, ist entweder die Definition der Hărte zu verwerfen, oder es muss eine der Voraussetzungen, unter denen die Formel (4) anwendbar ist, nicht erfüllt sein. Dazu gehört namentlich die, dass Platte und Linse aus gleichem Stoff bestehen, also insbesondere auch gleich hart sind; ist dies nicht der Fall, so muss man entweder nach einer complicirteren Formel aus der bekannten Härte des einen Körpers die des anderen auf Grund der Beobachtungen berechnen, oder man erhält, wenn man sich mit der Formel (4) begnügt, nur eine gewisse mittlere Härte von Platte und Linse. Nun bestanden zwar bei den hier behandelten Messungen Platte und Linse stets aus demselben Stoff, aber es ist sehr wohl denkbar, dass die Härte eines Körpers ausser von seinem Material auch von seiner Oberflächenkrümmung abhänge, und zwar offenbar in dem Sinne, dass die Härte desto grösser ist, je stärker die Krümmung ist. Eine experimentelle Bestätigung dieser Auf- 
fassung gibt der Umstand, dass bei richtiger und exacter Versuchsanordnung es immer die Platte ist, welche springt, und nicht die Linse. Je gekrummter also die benutzte Linse ist, desto härter würde sie hiernach sein, und desto grösser wurde auch die nach (4) gefundene mittlere Härte von Platte und Linse sich ergeben, wie es thatsächlich der Fall ist. Die Härte einer Linse würde sich hiernach durch eine Formel von der Gestalt:

$$
H=a+\frac{b}{\rho}
$$

darstellen, $a$ würde hierin die Härte einer ebenen Fläche desselben Materials, also gewissermaassen die "Eigenhärte" des Materials, $b$ dagegen die von der Krümmung abhängige "Oberflächenhärte" sein, also genau dem entsprechen, was man bei Flüssigkeiten „Oberflächenspannung“ nennt. ${ }^{2}$ )

Die zweite der erwähnten Vermuthungen läuft auf eine Modification der Hertz'schen Definition der Härte hinaus. Die nach ihr die Härte charakterisirende Grősse hat drei Kennzeichen, sie ist nämlich 1) ein Druck, sie hat 2) normale Richtung $\left(Z_{z}\right)$, und sie bezieht sich 3) auf den Mittelpunkt der Druckfläche. Gegen alle drei Punkte lassen sich Einwände erheben. Da nämlich bei spröden Körpern die charakteristische Erscheinung ein Sprung, also eine Trennung der Theile ist, so wird die unmittelbare Ursache nicht ein Druck, sondern ein Zug sein; da der Sprung von der Oberfläche $(z=0)$, wenn auch nicht senkrecht, so doch unter steilem Winkel ins Innere geht, so wird nicht $\boldsymbol{Z}_{\boldsymbol{z}}$, sondern ein schiefer Druck, annähernd sogar ein lateraler Druck $\left(X_{k}\right)$ in Betracht kommen; da endlich der Sprung den Rand der Druckfläche umgibt, so ist der Werth von $X_{x}$ an dieser Stelle einzuführen. Leider scheint dies bei der Complication der Formeln kaum durchführbar zu sein; aber so viel lässt sich erkennen, dass $X_{x}$ seinen grössten negativen Werth in der That etwas ausserhalb der Druckfläche erreicht, und

1) Eine andere Analogie ist die mit der Zugfestigkeit von Eisendrähten, welche nach Baumeister (Wied. Ann. 18. p. 578. 1883) desto grösser ist, je kleiner die Dicke des Drahtes ist, und zwar, wie ich finde, nach dem dem hier gefundenen gleichen Gesetze $F=$ const. $/ \sqrt[3]{d}$. 
ferner, dass dieser Maximalzug in anderer Weise von der Linsenkrümmung abbängen wird wie der Normaldruck, da letzterer offenbar von der Krümmung in beiden Dimensionen, jener aber nur von der Krümmung in einer Dimension beeinflusst wird; wenn also auch das Experiment für dasselbe Material je nach der Linsenkrümmung verschiedene Werthe von $\left(\boldsymbol{Z}_{s}\right)_{\max }$. liefert, so ist doch damit sehr wohl vereinbar, dass diesen verschiedenen Werthen ein und derselbe Werth von $\left(\boldsymbol{X}_{\boldsymbol{x}}\right)_{\max }$. entspricht. Vielleicht noch anschaulicher wird man sich das Gesagte vorstellen, wenu man bedenkt, dass der Maximaldruck auf die Druckfläche in dieser Fläche selbst ohne sichtbare Wirkung bleibt, diese Wirkung dagegen sich radial ausbreitet und in der Sprunglinie zum sichtbaren Ausdruck gelangt; man wird es dann verständlich finden, dass man, um gleiche Zahlen zu erhalten; $P$ nicht mit $D^{2}$ (Fläche), sondern mit einer geringeren Potenz von $D$ dividiren muss.

\section{\$ 8. Zahlenwerthe fuir Harte und Elasticität.}

Die zuletzt angestellten Betrachtungen lehren zwar, dass die theoretische Seite der Frage noch weiterer Ausfuhrung bedarf, die Lösung des Härteproblems selbst wird jedoch hierdurch kaum beeinträchtigt. Denn einmal war, wie bereits bemerkt, die Erlangung relativer Härtezahlen schon vor jenen Betrachtungen gesichert, sodann erhalten die Zahlen für einen bestimmten Fall ohne weiteres absolute Bedeutung, nämlich für den Fall, dass die drückende Linse den Krümmungsradius Eins hat, drittens und letztens aber wird die absolute Bedeutung der Zahlen sogar eine ganz allgemeine durch die Erwägung, dass, wenn die Multiplication der Hertz'schen Härte mit $\sqrt[3]{\varrho}$ für ein bestimmtes Material eine unter allen Umständen gleiche Zahl liefert, diese Zahl selbst dann als die absolute Härte des Materials bezeichnet werden darf, wenn ihre mechanische Bedeutung nicht vollständig oder mit Sicherheit bekannt ist - eine Einschränkung, welche auch noch fortfällt, sobald es gelingt, für die Existenz einer besonderen Obertlächenhärte auf anderem Wege den Beweis zu erbringen, den Maximalzug als Function der Linsenkrummung zu berechnen und das combinirte Ergebniss beider 
Punkte exact darzustellen. In diesem Sinne darf die Grösse $P_{1} \sqrt[3]{\varrho}$ als absolute Härte bezeichnet, also:

gesetzt werden.

$$
H=\frac{6}{\pi} \sqrt[3]{P \varrho q^{2}}
$$

Um ein Bild von den Schwankungen der gefundenen Härtezahlen zu geben, seien hier beispielsweise für zwei Versuchsreihen die - übrigens durchweg berechneten - Einzelwerthe angeführt.

$$
\begin{aligned}
& \text { Glas II. } \varrho=10 \text {. } \\
& \left.H=\begin{array}{llll}
220 & 230 & 222 & 218 \\
234 & 236 & 222 & 232
\end{array}\right\} 227 \pm 2 \\
& \text { Bergkrystall. } \varrho=1 \text {. } \\
& H=\begin{array}{lll|l}
298 & 281 & 299 & 292 \pm 2 \\
298 & 285 & 290 &
\end{array}
\end{aligned}
$$

\begin{tabular}{|c|c|c|c|c|c|c|c|c|c|}
\hline \multirow{2}{*}{ Stoff } & \multicolumn{8}{|c|}{$\rho=$} & \multirow{2}{*}{$\begin{array}{c}\text { Haupt- } \\
\text { mittel }\end{array}$} \\
\hline & 1 & 3 & 4 & 5 & 10 & 12 & 15 & 30 & \\
\hline Glas I & 212 & - & 215 & - & - & 214 & - & - & $214 \pm 1$ \\
\hline$"$ II & - & 228 & $\overline{04}$ & 222 & 227 & $\overline{297}$ & 223 & $\overline{296}$ & $226 \pm 2$ \\
\hline $\left.\begin{array}{c}\text { "III } \\
\text { Bergkrystall } \\
\text { senkr. z. Axe }\end{array}\right\}$ & 292 & - & $\begin{array}{l}244 \\
298\end{array}$ & - & - & $\begin{array}{l}237 \\
293\end{array}$ & - & $\begin{array}{l}236 \\
-\end{array}$ & $\begin{array}{l}258 \pm 2 \\
295 \pm 2\end{array}$ \\
\hline
\end{tabular}

Schliesslich erhält man die in folgender Tabelle zusam. mengestellten

Mittelwerthe der absoluten Härte.

Diese Zahlen weisen zunächst die Reihenfolge auf, welche nach den erwähnten Angaben uber die Glassorten, sowie nach der Stellung von Glas und Quarz in der Härtescala (Glas 4 bis 6, Quarz 7) zu erwarten war. Sie zeigen ferner eine für ein bisher noch nicht bearbeitetes Gebiet sehr befriedigende Genauigkeit von etwa 1 Proc., und es liegen z. B. selbst die extremsten, für benachbarte Glassorten gefundenen Werthe noch weit genug auseinander. Dass die Zabl für Quarz nicht noch erheblicher, als sie es thut, die Zahlen für Glas übertrifft, darf bei der nahen Verwandtschaft dieser Stoffe nicht $W$ under nehmen, und es sei hier die vorläufige Notiz hinzugefügt, dass, wie einige Vorversuche er- 
kennen lassen, parallel zur Axe liegende Quarzflächen sogar überhaupt nicht härter sind, als mittelhartes Glas.

Da die Härte nach unserer Definition nichts anderes ist, als eine bestimmte Art von Festigkeit, so liegt es nahe, die gefundenen Werthe mit anderen Arten von Festigkeit zu vergleichen. Für Glas ist dies, dank den Versuchen von v. Kowalski1), möglich; ich stelle daher die von ihm für thüringisches Glas gefundenen Werthe mit dem Mittelwerthe meiner Härtezahlen zusammen:

Festigkeit des Glases in $\mathbf{~ k g / q m m . ~}$

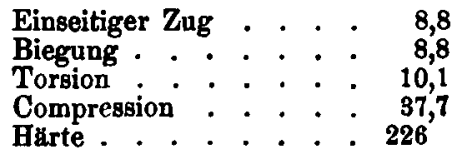

Wie man sieht, ist die Festigkeit gegen Biegung ebenso gross wie diejenige gegen Zug, dagegen ist die Festigkeit gegen Torsion etwas grösser, die gegen Compression viermal und endlich die Härte $26 \mathrm{mal}$ so gross.

Es bleibt noch übrig, nach Formel (5) die Elasticitätsconstante $E^{\prime}$ und daraus, wenn möglich, den Modul $E$ zu bestimmen. Für $E^{\prime}$ erhălt man ohne weiteres folgende Werthe:

$\begin{array}{ccccc}\text { Stoff } & \text { Glas I } & \text { Glas II } & \text { Glas III } & \text { Bergkrystall } \\ E^{\prime} & 5592 & 6960 & \mathbf{7 7 6 4} & 10164 \\ \text { W. Fehler } & \pm 15 & \pm 24 & \pm 45 & \pm 18\end{array}$

Die Constante $E^{\prime}$ setzt sich nun freilich aus den beiden Constanten $E$ und $\mu$ zusammen, und diese kann man einzeln bekanntlich erst durch Combination zweier Messungen, z. B. Längsdehnung und Quercontraction oder Biegung und Torsion, ermitteln; infolge der eigenthlumlichen Bedeutung von $E^{\prime}$ kann man jedoch auch ohne eine weitere Messung wenigstens ungefahre Werthe ron $E$ selbst angeben. Es ist dies dem Umstande zu verdanken, dass $\mu$ in der Form $\left(1-\mu^{2}\right)$ in $E^{\prime}$ enthalten ist, diese Function von $\mu$ aber nur sehr wenig sich

1) v. Kowalski, Unt. üb. d. Festigkeit d. Gluses. Leiprig (Göttingen) 1889. Wied. Ann. 36. p. 307. 1889. - Aeltere, die Zugfestigkeit betreffende Zablen von Wertheim sind betrichtlich kleiner.

Anv. d. Phys. a. Chem. N. F. XIIII. 
ändert, wenn für $\mu$ selbst die äussersten experimentell gefundenen Werthe eingesetzt werden.

Nach den Versuchen von Cornu ${ }^{1}$ ), Everett ${ }^{2}$ ), Voigt ${ }^{3}$ ), Canton $\left.e^{4}\right)$ und v. Kowalski ${ }^{5}$ ) liegt nämlich für Glas $\mu$ zwischen den Werthen 0,208 und 0,264, und diesen Extremen entsprechen für $\left(1-\mu^{2}\right)$ die Werthe 0,957 und 0,930 ; ferner wird der Mittelwerth und der wahrscheinliche Fehler von $\mu$ bei Berücksichtigung des Gewichtes der einzelnen Messungen:

und entsprechend:

$$
\mu=0,225 \pm 0,008
$$

$$
1-\mu^{2}=0,949 \pm 0,003 \text {. }
$$

Man würde also einen Fehler von nur $1 / 3$ Proc. zu erwarten haben, wenn nicht zu bedenken wäre, dass dieser Werth von $\mu$ nur für kleine Deformationen gilt, wăhrend er nach den Betruchtungen von Röntgen ${ }^{\theta}$ ) u. A. für grössere Deformationen kleiner ist; bei incompressibeln Körpern, bei denen für kleine Deformationen $\mu=0,5$ ist, ist diese $\mathrm{Ab}$ nahme sogar sehr beträchtlich; in unserem Falle wird sie viel unbedeutender sein; nach der Analogie kann man annehmen, dass man zu setzen hat:

$$
1-\mu^{2}=0,97 \pm 0,01 \text {, }
$$

wodurch der wahrscheinliche Fehler allerdings auf rund 1 Proc. gestiegen ist. Hiernach erhält man die Moduln der Glassorten, indem man die $E^{\prime}$ um 3 Proc. verkleinert; es wird also:

$\begin{array}{cccc}\text { Stoff } & \text { Glas I } & \text { Glas II } & \text { Glas III } \\ E & 5424 & 6751 & 7531\end{array}$

Die Elasticität verschiedener Glassorten ist hiernach eine recht erheblich verschiedene, und es ist daher bei der bisherigen Unbestimmtheit der Charakteristik der Glassorten eine eingehende Vergleichung mit den von anderen Beobachtern gefundenen Werthen nicht möglich. Dass sich je-

1) Cornu, Compt. rend. 69. p. 333. 1869.

2) Everett, Phil. Trans. 1867. p. 139.

3) Voigt, Wied. Aun, 15. p. 49i. 188\%.

4) Cantone, Rend. Acc. Linc. 4. p. 220 u. 292.1888.

5) v. Kowalski, l, c. p. 15.

6) Röntgen, Pogg. Ann. 1ō9. p. 601. 1876. 
doch obige Zahlen mit den ubrigen zwischen denselben Grenzen halten, zeigt folgende, auch an sich interessante Zusammenstellung:

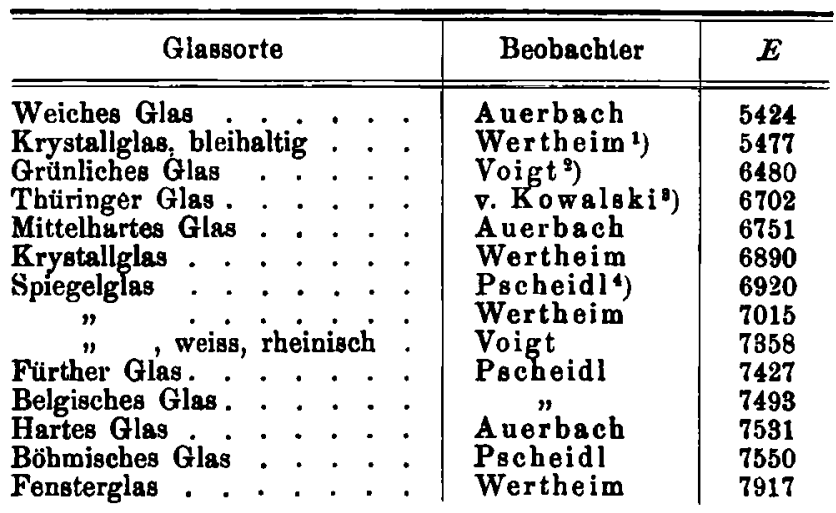

Es wird beabsichtigt, demnächst für eine grössere Anzahl genau charakterisirter Glassorten die Bestimmung von Elasticităt und Härte systematisch durchzuführen.

Für Bergkrystall hat die Grösse $\mu$ selbst keine Bedeu. tung; der Factor aber, mit welchem man $E^{\prime}$ multipliciren muss, um den Werth des Moduls in der Axenrichtung $E_{0}$ zu erhalten, ist hier mit Rücksicht auf das elastische Verhalten und die Härte dieses Materials jedenfalls noch năher an Eins gelegen, sodass der Fehler, den man begeht, wenn man geradezu $E_{0}=E^{\prime}$, also:

$$
E_{0}=10164
$$

setzt, jedenfalls innerhalb 2 Proc. bleiben wird. In der That ist dieser Werth gegenüber dem Voigt'schen (10304) nur um reichlich 1 Proc. kleiner - eine Differenz, welche in Anbetracht der so verschiedenen Ableitung schon an sich nicht sonderlich gross erscheinen wird, zum Theil aber vermuthlich sich dadurch erklärt, dass die Beanspruchung hier eine viel grössere ist als bei Voigt.

1) Wertheim u. Chevandier, Compt. rend. 20. p. 1637. 1845.

2) Voigt, Wied. Anu. 15. p. 497. 1882.

8) v. Kowalski, l, c. p. 10.

4) Pscheidl, Wien. Ber. 79. p. 114, 1877; 86. p. 115.1882. 
Von Interesse ist schliesslich noch eine Vergleichung der Härten mit den Elasticitätsmoduln. Wie man orkennt, ist von den untersuchten Stoffen der elastischere zwar auch der härtere, aber die Härte nimmt weniger stark zu als die Elasticitä; druckt man $H$ in Procenten von $E$ aus, so erhält man daher die abnehmenden Zahlen:

$\begin{array}{cccc}\text { Glas I } & \text { Glas II } & \text { Glas III } & \text { Bergkrystall } \\ \mathbf{3 , 9} & \mathbf{3 , 3} & \mathbf{3 , 2} & \mathbf{2 , 9}\end{array}$

Es macht sich das bei den Beobachtungen in charakteristischer und tberraschender Weise geltend; während man nämlich hätte erwarten sollen, dass man bei dem härteren Stoffe einen stärkeren Gesammtdruck würde ausuben mulssen, muss man dies im allgemeinen gerade bei dem weicheren thun, weil hier die Druckfläche sehr gross wird, also ein hoher Gesammtdruck erforderlich ist, um selbst nur einen mässigen Einheitsdruck zu erzielen.

Jena, Februar 1891. 


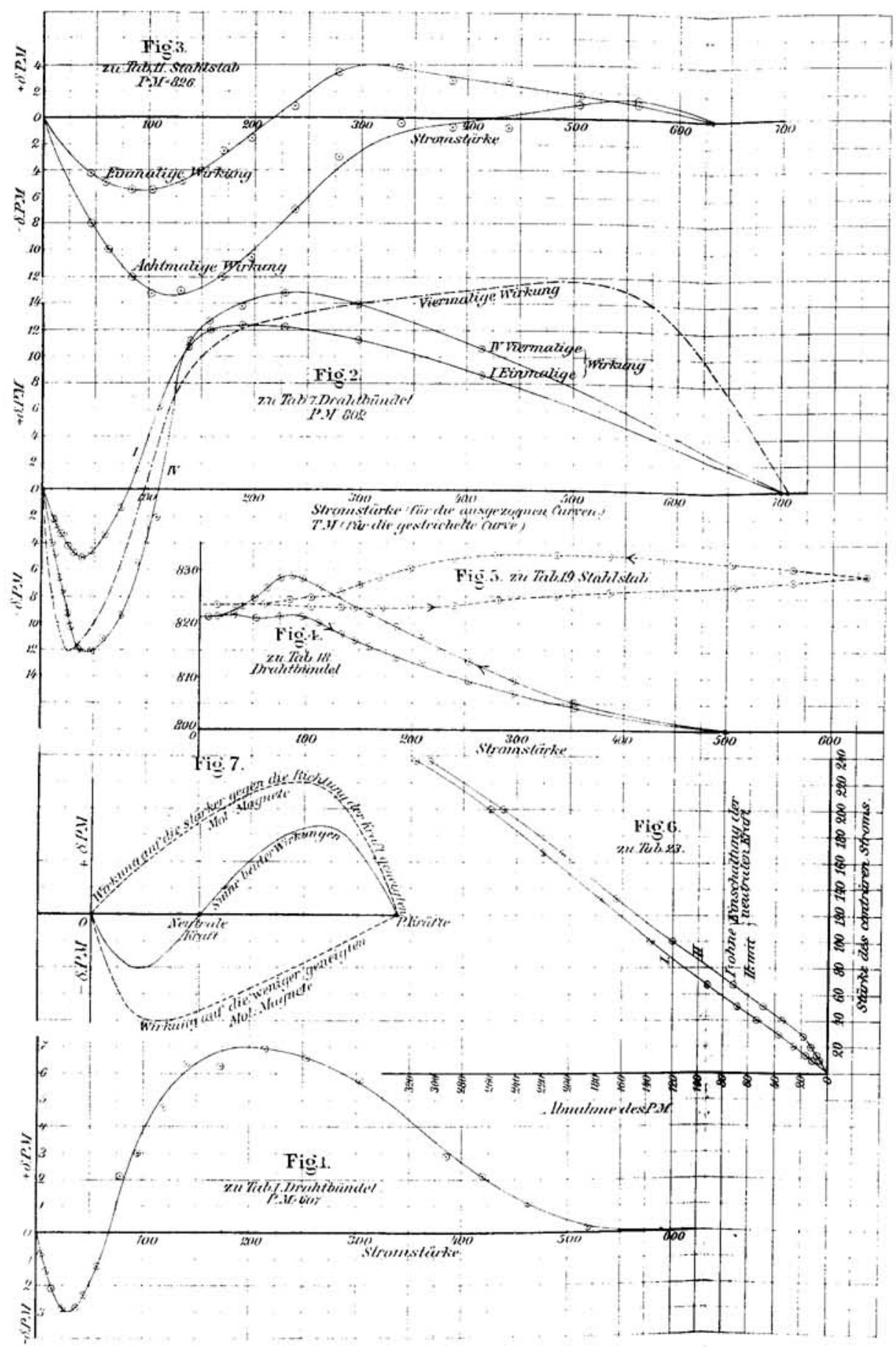

Fig..1.

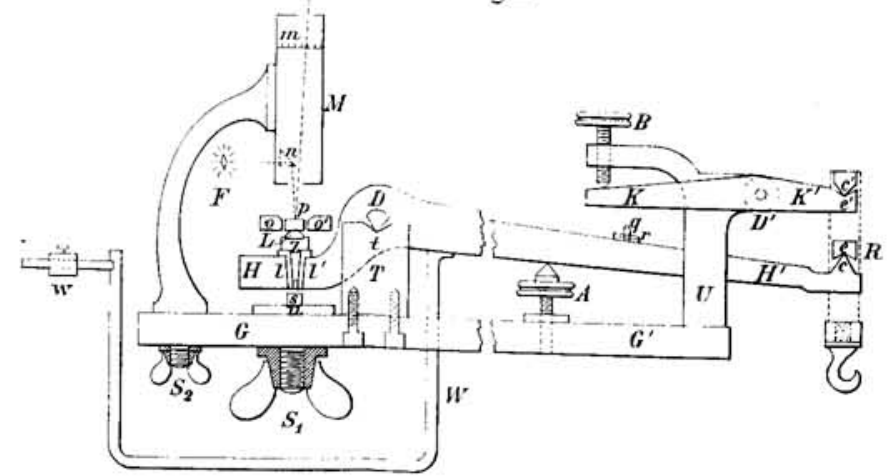

rig. 2.

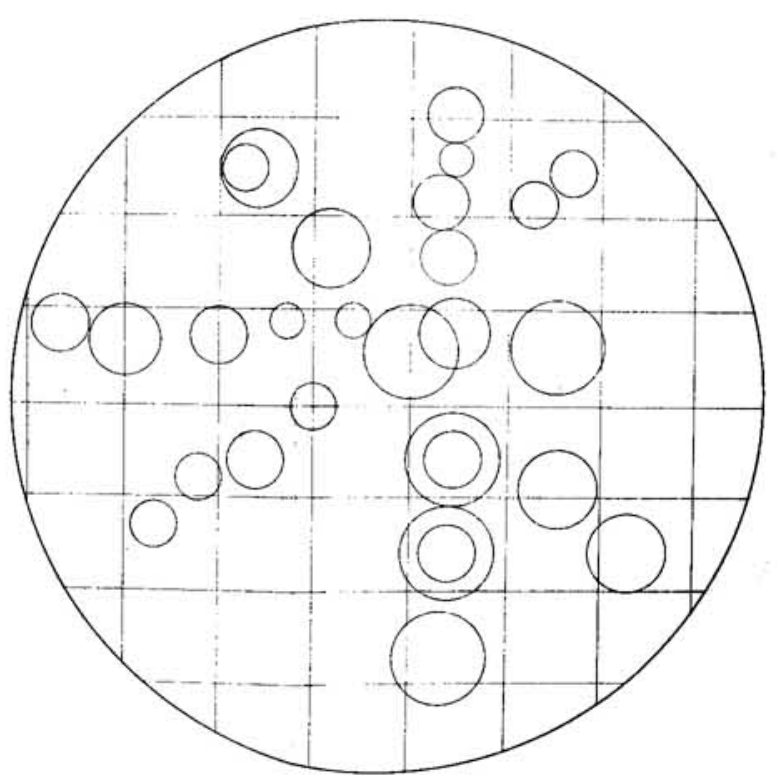

$x$ 